“C 2017 IEEE. Personal use of this material is permitted. Permission from IEEE must be obtained for all other uses, in any current or future media, including reprinting/republishing this material for advertising or promotional purposes, creating new collective works, for resale or redistribution to servers or lists, or reuse of any copyrighted component of this work in other works." 


\title{
Radio Frequency Self-interference Cancellation with Analog Least Mean Square Loop
}

\author{
Xiaojing Huang, Senior Member, IEEE and Y. Jay Guo, Fellow, IEEE
}

\begin{abstract}
A multi-tap adaptive filter with analog least mean square (ALMS) loop is proposed in this paper for effective and low complexity self-interference cancellation implemented as part of the radio frequency (RF) frontend in a full duplex transceiver. Comprehensive analyses of the ALMS loop's behaviors at both micro and macro scales are presented for a wireless communication system with single carrier signalling. It is revealed that there is always an irreducible residual interference due to the cyclostationary property of the transmitted signal. The interference suppression ratio (ISR) lower bound is derived accordingly, which can be used as a design rule for determining the ALMS loop parameter. Stationary analysis shows that the convergence speed and achievable ISR of the ALMS loop are determined by the loop gain and the autocorrelation function of the transmitted signal. The interference channel modelling error with the adaptive filter also accounts for part of the residual interference power. These theoretical findings are verified by simulation and experimental results.
\end{abstract}

Index Terms-Full duplex, adaptive filter, analog least mean square loop, and self-interference cancellation.

\section{INTRODUCTION}

Full duplex, i.e., transmission and reception at the same time in the same frequency band and in the same spatial location, has gained significant attention as a way to potentially double the spectral efficiency of future wireless communication systems [1-3]. It is also considered as a key technology for the emerging fifth generation $(5 \mathrm{G})$ mobile broadband networks [4,5]. Among the various challenging issues which need to be solved before the full duplex radio becomes a reality $[1,6]$, self-interference from the transmitter to the co-located receiver is the most serious and fundamental one.

A number of self-interference suppression and/or cancellation techniques have been reported in the literature, which can be divided into three categories: propagation (or antenna) domain suppression, analog domain cancellation, and digital domain cancellation [1]. A combination of them is often required to achieve the required level of self-interference suppression. Extensive research has also shown that the analog domain cancellation at radio frequency (RF) frontend, i.e., using the RF signal immediately before the transmitter antenna as the reference signal to cancel the self-interference received immediately after the receiver antenna, is the most effective approach. It can reduce the distortion due to practical impairments (such as nonlinearity) and relax the requirements for further downstream processing $[1,7,18]$.

Xiaojing Huang and Y. Jay Guo are with the Global Big Data Technologies Center, University of Technology Sydney, Australia (email: \{Xiaojing.Huang; Jay.Guo\}@uts.edu.au).
Many existing RF self-interference cancellation techniques can only cancel the direct path interference [2,7-11], whereas the others can cancel both the direct and reflected path interference [12-14]. Obviously, cancellation of all self-interference at RF frontend as much as possible is more desirable though it is more difficult especially for wideband systems.

The analog cancellation circuit with tuning algorithm employed at the RF frontend of a full duplex WiFi radio proposed in [12] is one of the notable full self-interference cancellation schemes published. The circuit consists of several parallel delay lines and tunable attenuators, each providing a copy of the transmitted signal. Multiple copies are combined to interpolate the self-interference which is then cancelled from the received signal. However, direct interpolation of an RF signal requires very finely determined delays (comparable to the inverse of the RF carrier frequency) and the number of delay lines may be quite large if the interference channel delay is long. The tunable attenuators also need to be dynamically determined by additional digitally implemented optimization algorithms. These lead to implementation complexity and hence limit its practical application.

The closed-loop self-interference cancellation circuit proposed in [13] provides a low complexity solution that can be implemented purely in analog domain. In this solution, the tapped delay lines are used together with phase shifters which provide orthogonal (quadrature) copies of the RF signal, and hence the delay between taps is comparable to the inverse of the transmitted signal bandwidth. The tap coefficients are determined by analog least mean square (ALMS) circuits implemented at baseband. However, as required by any conventional ALMS circuit [15-17], an ideal integrator is necessary, which has to be implemented by an active circuit. The baseband implementation for the tap weight control also requires additional down-conversion circuits and more analog multipliers. The experimental evaluations of similar canceller architectures are reported in [19, 21], which confirms that the ALMS based RF self-interference cancellation is very promising.

In this paper, we propose to use a multi-tap adaptive filter with simple ALMS loop all implemented at RF frontend for effective and low complexity self-interference cancellation in a full duplex radio. Instead of using active integrator circuits, we use simple passive resistor-capacitor (RC) circuits to reduce the hardware complexity. Unique to most existing RF self-interference cancellation techniques, including the above mentioned two typical ones by which the cancellation circuits are standalone entities, our ALMS loop incorporates the high power amplifier (HPA) and low noise amplifier (LNA) of 
the RF frontend to provide the necessary high loop gain. The behaviors of the ALMS loop are analyzed at both the micro scale, i.e., considering the cyclostationary property of the transmitted signal to examine the loop behaviors in a time scale of several data symbol durations, and the macro scale, i.e., treating the transmitted signal as a stationary process to assess the overall loop performance. The cyclostationary analysis at the micro scale reveals that there is always an irreducible residual interference due to the transmitted signal's cyclostational property, and hence a lower bound of the interference suppression ratio (ISR) is derived. This lower bound can be used as a design rule for determining the $\mathrm{RC}$ circuit parameters. Through stationary analysis at the macro scale, we also show that the ALMS loop performance is ultimately determined by the loop gain and the autocorrelation function of the transmitted signal in relation to the adaptive filter tap delay spacing. The residual interference power after cancellation is also found to be affected by the interference channel modelling error with the adaptive filter. The comprehensive analysis of the proposed ALMS loop establishes a solid theoretical foundation for RF self-interference cancellation research and development. For example, it can be used as a benchmark to study how to reduce the impact of in-phase/quadrature (I/Q) imbalance [20] on the ALMS loop performance.

The rest of this paper is organized as follows. In Section II, the RF self-interference cancellation structure with the proposed ALMS loop is introduced. In Section III, the convergence performance of the ALMS loop is analyzed for a single carrier system assuming the ideal cyclostationary property. The irreducible residual interference is revealed and an ISR lower bound is derived. In Section IV, stationary analysis of the ALMS loop with fractionally-spaced multi-tap filter for a general interference channel is performed to analyze the impact of the signal autocorrelation and interference channel modelling error on the loop convergence and residual interference power. Simulation and experimental results are given in Section V to verify the theoretical analysis. Finally, conclusions are drawn in Section VI.

\section{CANCEllation with ALMS Loop}

We first derive the RF self-interference cancellation structure with the proposed ALMS loop.

Consider a full duplex transceiver RF frontend as shown in Fig. 1, which includes an HPA and a transmitter antenna at the transmitting side, and a receiver antenna and an LNA at the receiving side. In practice, a single antenna can be also used for both transmitting and receiving with the transmitting path and the receiving path separated by a circulator. Denoting the RF signal at the input of the transmitter antenna as $x(t)$ and it's lowpass equivalent baseband signal as $X(t)$, we have $x(t)=\operatorname{Re}\left\{X(t) e^{j 2 \pi f_{c} t}\right\}$ where $f_{c}$ is the carrier frequency.

For a single carrier system, $X(t)$ can be expressed as

$$
X(t)=\sum_{i=-\infty}^{\infty} a_{i} p\left(t-i T_{s}\right)
$$

where $a_{i}$ is the complex data symbol, $T_{s}$ the symbol interval, and $p(t)$ the spectral shaping pulse. The complex data symbols

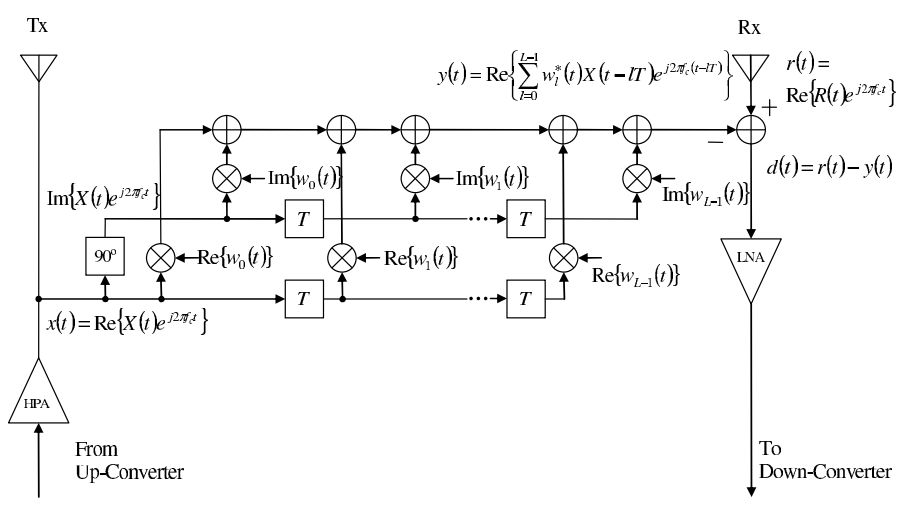

Fig. 1. RF frontend with multi-tap analog filter for self-interference cancellation.

are assumed to be independent of each other, satisfying $E\left\{a_{i}^{*} a_{i^{\prime}}\right\}=\left\{\begin{array}{ll}1, & \text { for } i=i^{\prime} \\ 0, & \text { for } i \neq i^{\prime}\end{array}\right.$ where $E\{\cdot\}$ denotes the ensemble expectation. The root mean square (RMS) value of $X(t)$ is defined as $V_{X}=\sqrt{\frac{1}{T_{s}} \int_{0}^{T_{s}} E\left\{|X(t)|^{2}\right\} d t}$ such that the average power of $X(t)$ with $1 \Omega$ load is $V_{X}^{2}$.

In order to cancel the self-interference at the receiver, a multi-tap analog filter is used to regenerate the selfinterference signal $y(t)$ and it is then subtracted from the received RF signal with transmitter self-interference at the output of the receiver antenna $r(t)=\operatorname{Re}\left\{R(t) e^{j 2 \pi f_{c} t}\right\}$ where $R(t)$ is the lowpass equivalent baseband signal of the received RF signal. The analog filter has $L$ stage taps with complex weighting coefficients $w_{l}(t), l=0,1, \cdots, L-1$, separated by a delay line with time delay $T$. Then the output signal of the analog filter can be expressed as

$$
y(t)=\operatorname{Re}\left\{\sum_{l=0}^{L-1} w_{l}^{*}(t) X(t-l T) e^{j 2 \pi f_{c}(t-l T)}\right\} .
$$

The structure of this multi-tap analog filter is shown in Fig. 1 , where the 90 degree phase shifter is used for providing the quadrature component of $x(t)$.

The design of the analog circuitry required for obtaining the weighting coefficients adaptively is discussed as follows. Analogous to the LMS algorithm in digital adaptive filtering, we would expect that these weighting coefficients should be obtained such that the instantaneous residual interference power after cancellation is minimized, i.e., $d^{2}(t)=[r(t)-y(t)]^{2} \rightarrow \min$, and this would result in the continuous-time LMS algorithm for updating the weighting coefficients [15]. Each weighting coefficient $w_{l}(t)$ would be obtained by integration over $-\frac{\partial d^{2}(t)}{\partial w_{l}(t)}$ and multiplying by a constant which determines the speed of convergence, where

$$
\begin{aligned}
\frac{\partial d^{2}(t)}{\partial w_{l}(t)} & =\frac{\partial d^{2}(t)}{\partial \operatorname{Re}\left\{w_{l}(t)\right\}}+j \frac{\partial d^{2}(t)}{\partial \operatorname{Im}\left\{w_{l}(t)\right\}} \\
& =-2[r(t)-y(t)] X(t-l T) e^{j 2 \pi f_{c}(t-l T)} .
\end{aligned}
$$

However, since an ideal analog integration circuit is difficult to implement in practice, we propose to use a more practical 
analog LMS circuit described by the differential equation

$$
\frac{d w_{l}(t)}{d t}+\alpha w_{l}(t)=-\frac{\mu \alpha}{K_{1} K_{2}} \frac{\partial d^{2}(t)}{\partial w_{l}(t)}, l=0,1, \cdots, L-1,
$$

where $\alpha, \mu, K_{1}$, and $K_{2}$ are constants which jointly determine the adaptation performance.

Eq. (4) represents the first-order lowpass filter (LPF) with transfer function $\frac{\alpha}{s+\alpha}$ (or impulse response $\alpha e^{-\alpha t}$ for $t>0$ ) and input signal $-\frac{\mu}{K_{1} K_{2}} \frac{\partial d^{2}(t)}{\partial w_{l}(t)}$. Such LPF is much easier to implement than an ideal integrator. For example, it can be a simple passive RC circuit. From (3) and (4), the weighting coefficient can be finally expressed as the convolution of the filter impulse response with the input signal, i.e.,

$$
\begin{array}{r}
w_{l}(t)=\frac{2 \mu \alpha}{K_{1} K_{2}} \int_{0}^{t} e^{-\alpha(t-\tau)}[r(\tau)-y(\tau)] \\
\cdot X(\tau-l T) e^{j 2 \pi f_{c}(\tau-l T)} d \tau,
\end{array}
$$

and thus the complete adaptive filter with ALMS loop can be implemented as shown in Fig. 2, where Fig. 2(a) is the structure which directly implements the adaptive filter shown in Fig. 1, Fig. 2(b) is an alternative structure which makes use of the I/Q modulation/demodulation architectures with less time delay elements, and Fig. 2(c) is the RC circuit for simple LPF implementation.

Note that the constants $K_{1}$ and $K_{2}$ are absorbed into respective multipliers in the adaptive filter for practical implementation. This is because any practical analog multiplier with two inputs $v_{i n 1}$ and $v_{i n 2}$ will produce an output $v_{\text {out }}=\frac{v_{i n 1} v_{i n 2}}{K}$ where $K$ is a dimensional constant. We assume that the multipliers in the I/Q demodulators have the same dimensional constant $K_{1}$ and the multipliers in the I/Q modulators have the same dimensional constant $K_{2}$. Thus, the outputs after LPFs in each I/Q demodulator will be $w_{l}(t) K_{2}$ and the output of the adaptive filter will be scaled by $\frac{1}{K_{1} K_{2}}$ in total. Also note that the LNA is incorporated into the ALMS loop to provide the feedback loop gain $2 \mu$.

\section{Cyclostationary AnAlysis}

We then proceed to analyze the ALMS loop at micro scale, derive the performance bound, and discuss how to select the loop parameter for achieving the required performance.

To start with, let's revisit an important property of the transmitted signal $X(t)$ in terms of its autocorrelation function defined as $\Phi_{X X}(t ; \tau)=E\left\{X^{*}(t) X(t-\tau)\right\}$. From (1) we have

$$
\begin{aligned}
& \Phi_{X X}(t ; \tau) \\
& =\sum_{i=-\infty}^{\infty} \sum_{i^{\prime}=-\infty}^{\infty} E\left\{a_{i}^{*} a_{i^{\prime}}\right\} p^{*}\left(t-i T_{s}\right) p\left(t-\tau-i^{\prime} T_{s}\right) \\
& =\sum_{i=-\infty}^{\infty} p^{*}\left(t-i T_{s}\right) p\left(t-\tau-i T_{s}\right) .
\end{aligned}
$$

It is seen that $\Phi_{X X}(t ; \tau)$ satisfies the property

$$
\Phi_{X X}(t ; \tau)=\Phi_{X X}\left(t+T_{s} ; \tau\right), \text { for all } t \text { and } \tau
$$

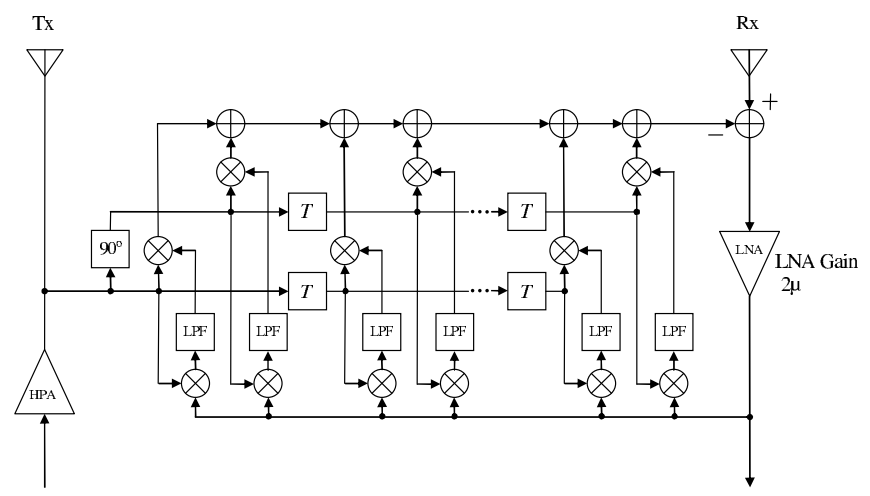

(a)

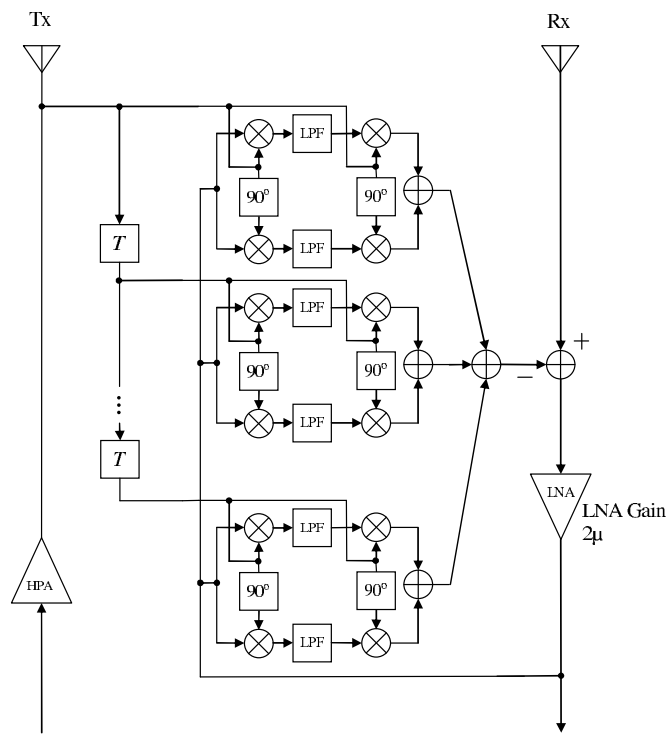

(b)

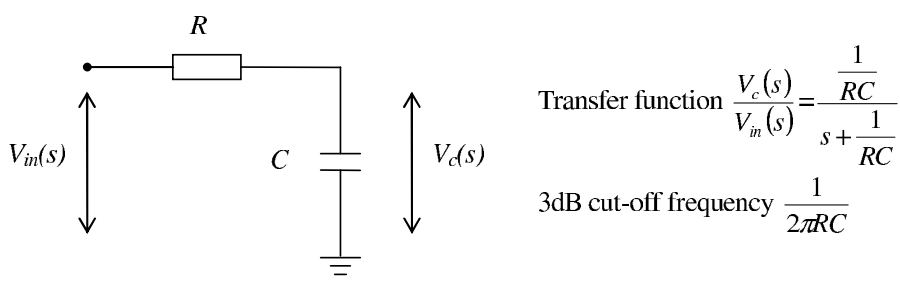

(c)

Fig. 2. (a) Complete adaptive filter with ALMS loop; (b) Alternative adaptive filter structure; and (c) RC lowpass filter.

which means that $X(t)$ is wide-sense cyclostationary. This cyclostationary property has a fundamental impact on how much the self-interference cancellation can be ultimately achieved.

\section{A. Weighting Coefficient Estimation Error Modelling}

Suppose that the received RF signal can be expressed as

$$
r(t)=z(t)+s(t)+n(t)
$$

where $z(t)$ is the self-interference from the local transmitter, $s(t)$ the received information bearing RF signal from the 
remote transmitter, and $n(t)$ the additive Gaussian noise at the receiver. Their lowpass equivalent baseband versions are denoted as $Z(t), S(t)$, and $N(t)$, respectively. For simplicity, we assume that the the interference channel can be modelled as a tapped delay line filter so that $Z(t)$ can be expressed as

$$
Z(t)=\sum_{l=0}^{L-1} h_{l}^{*} X\left(t-l T_{s}\right)
$$

where $h_{l}^{*}, l=0,1, \cdots, L-1$, are the tap coefficients and $L$ is the number of taps. A general interference channel will be considered in the next section and the modelling error will be discussed accordingly.

We also assume that the time delay for each delay element in the adaptive filter is the same as the data symbol interval $T_{s}$ and the number of taps is known, which is the same as that in the interference model (9).

Substituting (2), (8), and (9) into (5), we obtain $w_{l}(t)$ as shown in (10) on top of next page. In deriving (10), we have assumed that the bandwidth of the LPF is very narrow relative to $f_{c}$ so that the signal components centred about frequency $2 f_{c}$ are all eliminated after lowpass filtering. After some further manipulation, Eq. (10) can be expressed as

$$
\begin{array}{r}
u_{l}(t)=h_{l}-\frac{\mu \alpha}{K_{1} K_{2}} \int_{0}^{t} e^{-\alpha(t-\tau)}\left[\sum_{l^{\prime}=0}^{L-1} u_{l^{\prime}}(\tau) X^{*}\left(\tau-l^{\prime} T_{s}\right)+\right. \\
\left.S^{*}(\tau)+N^{*}(\tau)\right] X\left(\tau-l T_{s}\right) d \tau
\end{array}
$$

where

$$
u_{l}(t)=h_{l}-w_{l}(t) e^{j 2 \pi f_{c} T_{s} l}
$$

is the error between the $l$-th modelled tap coefficient of the interference channel and the weight of the $l$-th tap of the adaptive filter with a phase shift $2 \pi f_{c} T_{s} l$.

Taking the expectation over (11) and assuming that $u_{l}(t)$, $S(t), N(t)$, and $X(t)$ are independent of each other, we have

$\bar{u}_{l}(t)$

$$
=h_{l}-\frac{\mu \alpha}{K_{1} K_{2}} \int_{0}^{t} e^{-\alpha(t-\tau)} \sum_{l^{\prime}=0}^{L-1} \bar{u}_{l^{\prime}}(\tau) \Phi_{X X}\left(\tau ;\left(l-l^{\prime}\right) T_{s}\right) d \tau
$$

$l=0,1, \cdots, L-1$,

where $\bar{u}_{l}(t)=E\left\{u_{l}(t)\right\}$ is the expected value of $u_{l}(t)$. Note that the independence assumption between $u_{l}(t)$ and $X(t)$ is made to simplify the mathematical manipulation though they are, strictly speaking, correlated. Nevertheless, the set of first order integral equations expressed in Eq. (13) still well describes the micro scale behaviours of the average weight estimation error in the ALMS loop when the transmitted signal is cyclostationary, as will be seen from the following analysis and the numerical simulation results.

\section{B. Solution under Ideal Autocorrelation Model}

Solving the above set of integral equations is extremely difficult if it is not impossible. To simplify the analysis and derive a useful performance bound, let's have a close look of $\Phi_{X X}(t ; \tau)$ for the popular baseband transmission system

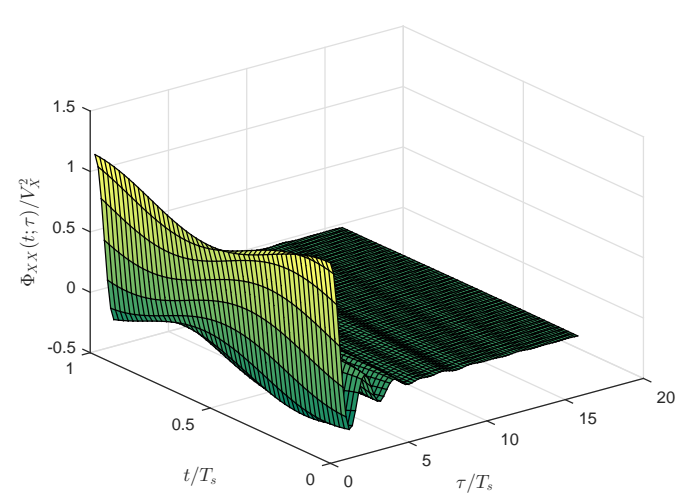

(a)

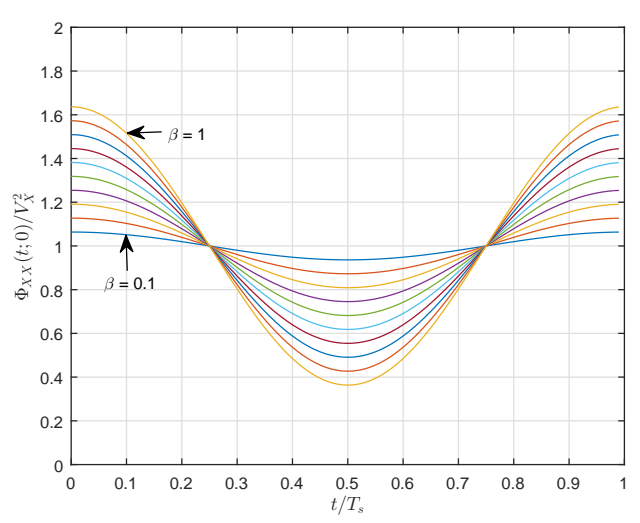

(b)

Fig. 3. (a) Normalized autocorrelation function $\frac{\Phi_{X X}(t ; \tau)}{V_{X}^{2}}$ with roll-off factor $\beta=0.25$; (b) Normalized autocorrelation function $\frac{\Phi_{X X}(t ; 0)}{V_{X}^{2}}$ with roll-off factor $\beta$ from 0.1 to 1 .

with root raised cosine (RRC) pulse shaping. Fig. 3 (a) shows the normalized autocorrelation function $\Phi_{X X}(t ; \tau) / V_{X}^{2}$ calculated by (6) using RRC with roll-off factor $\beta=0.25$. Since the autocorrelation function is periodic with respect to time variable $t$, only one period is shown. With respect to the time offset $\tau$, it is symmetric and decays rapidly. Fig. 3(b) shows the normalized autocorrelation function with time offset $\tau=0$ under various roll-off factor values. From this figure, we see that $\Phi_{X X}(t ; 0)$ can be well approximated as $V_{X}^{2}\left(\frac{2 \beta}{\pi} \cos \frac{2 \pi}{T_{s}} t+1\right)$.

Based on the autocorrelation approximation at zero time offset and further assuming that it is zero at any time offset which is an integer multiple of $T_{s}$, we can model the ideal autocorrelation function as

$\Phi_{X X}\left(t ;\left(l-l^{\prime}\right) T_{s}\right)=\left\{\begin{array}{ll}V_{X}^{2}\left(\frac{2 \beta}{\pi} \cos \frac{2 \pi}{T_{s}} t+1\right), & \text { for } l=l^{\prime} \\ 0, & \text { for } l \neq l^{\prime}\end{array}\right.$.

Then, by defining

$$
A^{2}=\frac{V_{X}^{2}}{K_{1} K_{2}}
$$




$$
\begin{aligned}
& w_{l}(t) \\
& =\frac{2 \mu \alpha}{K_{1} K_{2}} \int_{0}^{t} e^{-\alpha(t-\tau)} R e\left\{\left[\sum_{l^{\prime}=0}^{L-1}\left[h_{l^{\prime}}-w_{l^{\prime}}(\tau) e^{j 2 \pi f_{c} T_{s} l^{\prime}}\right]^{*} X\left(\tau-l^{\prime} T_{s}\right)+S(\tau)+N(\tau)\right] e^{j 2 \pi f_{c} \tau}\right\} X\left(\tau-l T_{s}\right) e^{j 2 \pi f_{c}\left(\tau-l T_{s}\right)} d \tau \\
& =\frac{\mu \alpha}{K_{1} K_{2}} \int_{0}^{t} e^{-\alpha(t-\tau)}\left\{\left[\sum_{l^{\prime}=0}^{L-1}\left[h_{l^{\prime}}-w_{l^{\prime}}(\tau) e^{j 2 \pi f_{c} T_{s} l^{\prime}}\right]^{*} X\left(\tau-l^{\prime} T_{s}\right)+S(\tau)+N(\tau)\right] e^{j 2 \pi f_{c} \tau}+\right. \\
& \left.\quad\left[\sum_{l^{\prime}=0}^{L-1}\left[h_{l^{\prime}}-w_{l^{\prime}}(\tau) e^{j 2 \pi f_{c} T_{s} l^{\prime}}\right] X^{*}\left(\tau-l^{\prime} T_{s}\right)+S^{*}(\tau)+N^{*}(\tau)\right] e^{-j 2 \pi f_{c} \tau}\right\} X\left(\tau-l T_{s}\right) e^{j 2 \pi f_{c}\left(\tau-l T_{s}\right)} d \tau \\
& =\frac{\mu \alpha}{K_{1} K_{2}} \int_{0}^{t} e^{-\alpha(t-\tau)}\left[\sum_{l^{\prime}=0}^{L-1}\left[h_{l^{\prime}}-w_{l^{\prime}}(\tau) e^{j 2 \pi f_{c} T_{s} l^{\prime}}\right] X^{*}\left(\tau-l^{\prime} T_{s}\right)+S^{*}(\tau)+N^{*}(\tau)\right] X\left(\tau-l T_{s}\right) e^{-j 2 \pi f_{c} T_{s} l} d \tau
\end{aligned}
$$

Eq. (13) can be simplified as

$\bar{u}_{l}(t)=h_{l}-\mu A^{2} \alpha \int_{0}^{t} e^{-\alpha(t-\tau)}\left(\frac{2 \beta}{\pi} \cos \frac{2 \pi}{T_{s}} \tau+1\right) \bar{u}_{l}(\tau) d \tau$

which is a Volterra integral equation of the second kind. A closed-form approximate solution is then found as (see Appendix A)

$$
\bar{u}_{l}(t) \approx h_{l} \frac{1+\mu A^{2} e^{-\left(1+\mu A^{2}\right) \alpha t}}{1+\mu A^{2}} e^{-\mu A^{2} \alpha T_{s} \frac{\beta}{\pi^{2}} \sin \frac{2 \pi}{T_{s}} t} .
$$

The term $e^{-\mu A^{2} \alpha T_{s} \frac{\beta}{\pi^{2}} \sin \frac{2 \pi}{T_{s}} t}$ is a periodic multiplicative function which represents the impact of the cyclostationary property. That is, each tap weighting coefficient in the ALMS loop never converges to a steady value but always changes with time. The variation between $\bar{u}_{l}(t)$ and the expected weight error without cyclostationary behavior, i.e., $h_{l} \frac{1+\mu A^{2} e^{-\left(1+\mu A^{2}\right) \alpha t}}{1+\mu A^{2}}$, can be expressed as

$$
\tilde{u}_{l}(t)=h_{l} \frac{1+\mu A^{2} e^{-\left(1+\mu A^{2}\right) \alpha t}}{1+\mu A^{2}}\left(e^{-\mu A^{2} \alpha T_{s} \frac{\beta}{\pi^{2}} \sin \frac{2 \pi}{T_{s}} t}-1\right) .
$$

Some plots of the normalized $\bar{u}_{l}(t)$, i.e., $\frac{\bar{u}_{l}(t)}{h_{l}}$, for $\beta=0.25$ are shown in Fig. 4(a) with different parameters $\alpha, \mu$ and $A$. The corresponding plots of the normalized variation, i.e., $\frac{\tilde{u}_{l}(t)}{h_{l}}$, are also shown in Fig. 4(b).

\section{Discussions}

From (17), (18), and Fig. 4, we can make the following observations:

1) When $t \rightarrow \infty, \quad \bar{u}_{l}(t)$ and $\tilde{u}_{l}(t)$ will converge to $h_{l} \frac{1}{1+\mu A^{2}} e^{-\mu A^{2} \alpha T_{s} \frac{\beta}{\pi^{2}} \sin \frac{2 \pi}{T_{s}} t} \quad$ and $h_{l} \frac{1}{1+\mu A^{2}}\left(e^{-\mu A^{2} \alpha T_{s} \frac{\beta}{\pi^{2}} \sin \frac{2 \pi}{T_{s}} t}-1\right)$ respectively. Both become periodic functions of $t$;

2) The speed of convergence is jointly determined by the parameters $\alpha, \mu$ and $A$, i.e., the factor $\left(1+\mu A^{2}\right) \alpha$ as it appears in the exponential function $e^{-\left(1+\mu A^{2}\right) \alpha t}$;

3) Ignoring the cyclostationary effect, the weight error is $h_{l} \frac{1}{1+\mu A^{2}}$ which is determined by the loop gain $\mu A^{2}$. This error means that the interference can not be completely cancelled by the ALMS loop and some

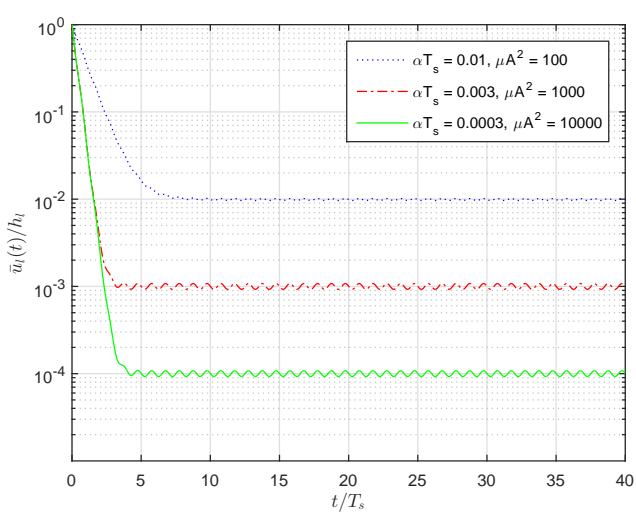

(a)

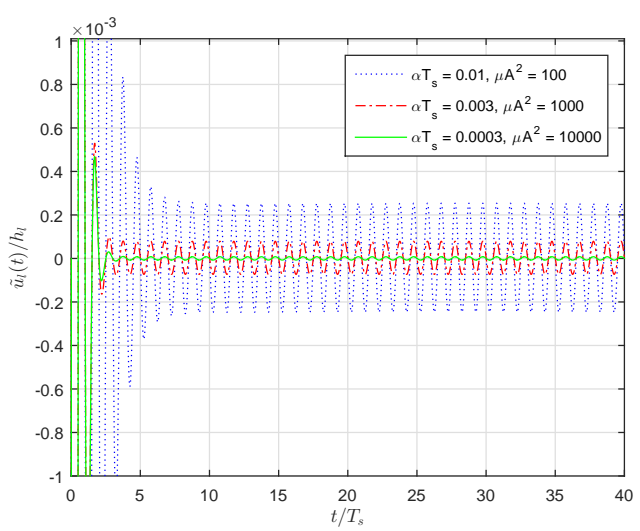

(b)

Fig. 4. (a) Normalized weight error; and (b) Normalized weight error variation.

residual interference remains. The average residual interference power (normalized by $K_{1} K_{2}$ ) can be evaluated as $P_{R I}=\frac{1}{\left(1+\mu A^{2}\right)^{2}} \frac{A^{2}}{2} \sum_{l=0}^{L-1}\left|h_{l}\right|^{2}$. Since the average normalized interference power is $P_{I}=\frac{A^{2}}{2} \sum_{l=0}^{L-1}\left|h_{l}\right|^{2}$, we obtain the interference suppression ratio as $I S R=$ $\frac{P_{R I}}{P_{I}}=\frac{1}{\left(1+\mu A^{2}\right)^{2}}$

4) Further cancellation can be achieved by digital domain methods to be performed at digital baseband. However, due to the weight variation caused by the cyclostation- 


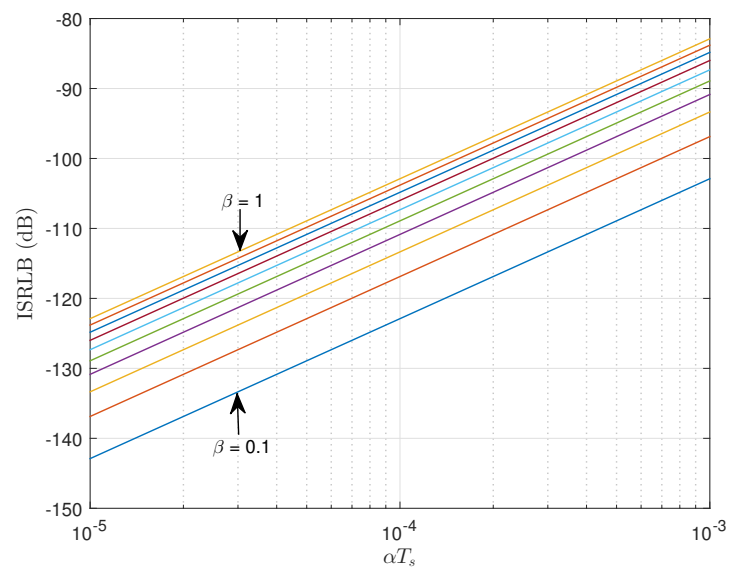

Fig. 5. Relationship between $I S R L B$ and $\alpha T_{s}$ for various RRC roll-off factors.

ary property, the residual interference cannot be totally cancelled. If $\mu A^{2}$ is sufficiently large, the power of this irreducible interference can be evaluated as

$$
\begin{aligned}
P_{I I} & =\frac{A^{2}}{2} \sum_{l=0}^{L-1} \frac{1}{T_{s}} \int_{0}^{T_{s}}\left|\tilde{u}_{l}(t)\right|^{2} d t \\
& =\frac{A^{2}}{2} \sum_{l=0}^{L-1}\left|h_{l}\right|^{2} \\
& \cdot \frac{1}{T_{s}} \int_{0}^{T_{s}}\left[\frac{1}{1+\mu A^{2}}\left(e^{-\mu A^{2} \alpha T_{s} \frac{\beta}{\pi^{2}} \sin \frac{2 \pi}{T_{s}} t}-1\right)\right]^{2} d t \\
& \approx P_{I} \frac{1}{T_{s}} \int_{0}^{T_{s}}\left(\frac{\mu A^{2}}{1+\mu A^{2}} \alpha T_{s} \frac{\beta}{\pi^{2}} \sin \frac{2 \pi}{T_{s}} t\right)^{2} d t \\
& \approx P_{I}\left(\alpha T_{s} \frac{\beta}{\pi^{2}}\right)^{2} \frac{1}{T_{s}} \int_{0}^{T_{s}} \sin ^{2} \frac{2 \pi}{T_{s}} t d t \\
& =\frac{1}{2} P_{I}\left(\alpha T_{s} \frac{\beta}{\pi^{2}}\right)^{2}
\end{aligned}
$$

and thus an ISR lower bound is

$$
I S R L B=\frac{P_{I I}}{P_{I}}=\frac{1}{2}\left(\alpha T_{s} \frac{\beta}{\pi^{2}}\right)^{2}
$$

which is determined by the lowpass filter RC constant (or the bandwidth) since $\alpha=\frac{1}{R C}$. The ISRLB represents the lowest possible interference suppression ratio achieved by both analog and digital means. From (20), the relationship between $I S R L B$ and $\alpha T_{s}$ is also shown in Fig. 5 for various RRC roll-off factors.

Observation 3) and 4) provide us useful guidelines for the designing process of the ALMS loop for RF self-interference cancelation with predicted performance. The process can be summarized as: 1. determining the loop gain $\mu A^{2}$ according to the desired ISR at RF stage; and 2. selecting the lowpass filter $\mathrm{RC}$ constant according to the overall interference cancellation performance requirement to be achieved by both analog and digital cancellations.

\section{Stationary Analysis}

When the LPF parameter is appropriately selected such that the $I S R L B$ is significantly lower than the ISR achieved by the RF ALMS loop, the impact of the cyclostationary property can be ignored, that is, the transmitted signal can be treated as a stationary process. The convergence behaviors of the weight and the residual interference power can then be analyzed at macro scale using stationary analysis. The non-ideal signal autocorrelation, fractionally-spaced tap delay in the analog adaptive filter, and a general interference channel can all be incorporated in this analysis.

For stationary analysis, both ensemble expectation and time averaging are used, and the combined operation is denoted by the operator $\bar{E}\{\cdot\}$. The normalized autocorrelation function is then expressed as

$$
\begin{aligned}
\Phi(\tau) & =\frac{1}{K_{1} K_{2}} \bar{E}\left\{X^{*}(t) X(t-\tau)\right\} \\
& =\frac{1}{K_{1} K_{2} T_{s}} \int_{0}^{T_{s}} E\left\{X^{*}(t) X(t-\tau)\right\} d t \\
& =\frac{1}{K_{1} K_{2} T_{s}} \int_{0}^{T_{s}} \Phi_{X X}(t ; \tau) d t \\
& =\frac{1}{K_{1} K_{2} T_{s}} \int_{-\infty}^{\infty} p^{*}(t) p(t-\tau) d t .
\end{aligned}
$$

\section{A. Interference Channel Modelling}

Let's model a general interference channel $h(t)$ as an $L$-stage tapped delay line filter with any fractionally-spaced tap delay $T$, i.e., $Z(t)=\int_{-\infty}^{\infty} h^{*}(\tau) X(t-\tau) d \tau \approx$ $\sum_{l=0}^{L-1} h_{l}^{*} X(t-l T)$. The modelled tap coefficients $h_{l}, \quad l=0,1, \cdots, L-1$, can be obtained through minimizing the normalized modelling error, i.e., $\varepsilon^{2}=\frac{1}{K_{1} K_{2}} \bar{E}\left\{\left|Z(t)-\sum_{l=0}^{L-1} h_{l}^{*} X(t-l T)\right|^{2}\right\}=\frac{1}{K_{1} K_{2}}$ $\bar{E}\left\{\left|\int_{-\infty}^{\infty} h^{*}(\tau) X(t-\tau) d \tau-\sum_{l=0}^{L-1} h_{l}^{*} X(t-l T)\right|^{2}\right\} \rightarrow$ min. Applying the principal of orthogonality, i.e.,

$$
\begin{aligned}
& \bar{E}\left\{\left[Z^{*}(t)-\sum_{l^{\prime}=0}^{L-1} h_{l^{\prime}} X^{*}\left(t-l^{\prime} T\right)\right] X(t-l T)\right\}=0, \\
& l=0,1, \cdots, L-1,
\end{aligned}
$$

we obtain the modelled tap coefficients

$$
\begin{aligned}
\mathbf{h} & =\left[\begin{array}{c}
h_{0} \\
h_{1} \\
\vdots \\
h_{L-1}
\end{array}\right] \\
& =\Phi^{-1}\left(\begin{array}{c}
\int_{-\infty}^{\infty} h(\tau) \Phi(-\tau) d \tau \\
\int_{-\infty}^{\infty} h(\tau) \Phi(T-\tau) d \tau \\
\vdots \\
\int_{-\infty}^{\infty} h(\tau) \Phi((L-1) T-\tau) d \tau
\end{array}\right)
\end{aligned}
$$

and the modelling error as shown in (24) on top of next page where $(\cdot)^{H}$ denotes conjugation and transposition, $\int_{-\infty}^{\infty} \int_{-\infty}^{\infty} h^{*}(\tau) h\left(\tau^{\prime}\right) \Phi\left(\tau-\tau^{\prime}\right) d \tau^{\prime} d \tau$ is the total normalized interference power at baseband, and $\Phi=$ $\left[\begin{array}{cccc}\Phi(0) & \Phi(-T) & \cdots & \Phi(-(L-1) T) \\ \Phi(T) & \Phi(0) & \cdots & \Phi(-(L-2) T) \\ \vdots & \vdots & \ddots & \vdots \\ \Phi((L-1) T) & \Phi((L-2) T) & \cdots & \Phi(0)\end{array}\right]$ is the 


$$
\begin{aligned}
\varepsilon^{2} & =\frac{1}{K_{1} K_{2}} \bar{E}\left\{\left[\int_{-\infty}^{\infty} h^{*}\left(\tau^{\prime}\right) X\left(t-\tau^{\prime}\right) d \tau^{\prime}-\sum_{l^{\prime}=0}^{L-1} h_{l^{\prime}}^{*} X\left(t-l^{\prime} T\right)\right]^{*}\left[\int_{-\infty}^{\infty} h^{*}(\tau) X(t-\tau) d \tau-\sum_{l=0}^{L-1} h_{l}^{*} X(t-l T)\right]\right\} \\
& =\frac{1}{K_{1} K_{2}} \bar{E}\left\{\left[\int_{-\infty}^{\infty} h\left(\tau^{\prime}\right) X^{*}\left(t-\tau^{\prime}\right) d \tau^{\prime}-\sum_{l^{\prime}=0}^{L-1} h_{l^{\prime}} X^{*}\left(t-l^{\prime} T\right)\right]^{*} \int_{-\infty}^{\infty} h^{*}(\tau) X(t-\tau) d \tau\right\} \\
& =\int_{-\infty}^{\infty} \int_{-\infty}^{\infty} h^{*}(\tau) h\left(\tau^{\prime}\right) \Phi\left(\tau-\tau^{\prime}\right) d \tau^{\prime} d \tau-\sum_{l^{\prime}=0}^{L-1} h_{l^{\prime}} \int_{-\infty}^{\infty} h^{*}(\tau) \Phi\left(\tau-l^{\prime} T\right) d \tau \\
& =\int_{-\infty}^{\infty} \int_{-\infty}^{\infty} h^{*}(\tau) h\left(\tau^{\prime}\right) \Phi\left(\tau-\tau^{\prime}\right) d \tau^{\prime} d \tau-\left(\begin{array}{c}
\int_{-\infty}^{\infty} h(\tau) \Phi(T-\tau) d \tau \\
\vdots \\
\int_{-\infty}^{\infty} h(\tau) \Phi((L-1) T-\tau) d \tau
\end{array}\right) \mathbf{h} \\
& =\int_{-\infty}^{\infty} \int_{-\infty}^{\infty} h^{*}(\tau) h\left(\tau^{\prime}\right) \Phi\left(\tau-\tau^{\prime}\right) d \tau^{\prime} d \tau-\mathbf{h}^{H} \mathbf{\Phi} \mathbf{h}
\end{aligned}
$$

\section{B. Convergence of Weighting Coefficients}

Following a similar process as shown in (10) and (11) but with a general interference channel and any fractionally-spaced time delay $T$ between taps of the analog adaptive filter, we have

$$
\begin{aligned}
& u_{l}(t)= \\
& h_{l}-\frac{\mu \alpha}{K_{1} K_{2}} \int_{0}^{t} e^{-\alpha(t-\tau)}\left[Z^{*}(\tau)-\sum_{l^{\prime}=0}^{L-1} h_{l^{\prime}} X^{*}\left(\tau-l^{\prime} T\right)+\right. \\
& \left.\sum_{l^{\prime}=0}^{L-1} u_{l^{\prime}}(\tau) X^{*}\left(\tau-l^{\prime} T\right)+S^{*}(\tau)+N^{*}(\tau)\right] X(\tau-l T) d \tau .
\end{aligned}
$$

Performing both ensemble expectation and time averaging, applying the principal of orthogonality (22), and making the same independence assumption as before, the stationary form of the integral equation (13) becomes

$$
\overline{\bar{u}}_{l}(t)=h_{l}-\mu \alpha \int_{0}^{t} e^{-\alpha(t-\tau)} \sum_{l^{\prime}=0}^{L-1} \overline{\bar{u}}_{l^{\prime}}(\tau) \Phi\left(\left(l-l^{\prime}\right) T\right) d \tau,
$$

or, in matrix form

$$
\overline{\overline{\mathbf{u}}}(t)=\mathbf{h}-\mu \alpha \int_{0}^{t} e^{-\alpha(t-\tau)} \mathbf{\Phi} \overline{\overline{\mathbf{u}}}(t) d \tau
$$

where $\overline{\overline{\mathbf{u}}}(t)=\left[\begin{array}{c}\overline{\bar{u}}_{0}(t) \\ \overline{\bar{u}}_{1}(t) \\ \vdots \\ \overline{\bar{u}}_{L-1}(t)\end{array}\right]$ and $\overline{\bar{u}}_{l}(t)=\bar{E}\left\{u_{l}(t)\right\}$.

Solving the above set of integral equations directly is still difficult. However, since $\boldsymbol{\Phi}$ can be decomposed as $\mathbf{\Phi}=\mathbf{Q} \boldsymbol{\Lambda} \mathbf{Q}^{-1}$ where $\mathbf{Q}$ is the orthonormal modal matrix whose columns are the $L$ eigenvectors of $\Phi$ and $\Lambda=$ $\left[\begin{array}{cccc}\lambda_{0} & 0 & \cdots & 0 \\ 0 & \lambda_{1} & \cdots & 0 \\ \vdots & \vdots & \ddots & \vdots \\ 0 & 0 & \cdots & \lambda_{L-1}\end{array}\right]$ is the spectral matrix whose main diagonal elements are the $L$ eigenvalues of $\boldsymbol{\Phi}$, Eq. (26) can be rewritten as

$$
\overline{\overline{\mathbf{v}}}(t)=\mathbf{g}-\mu \alpha \int_{0}^{t} e^{-\alpha(t-\tau)} \boldsymbol{\Lambda} \overline{\overline{\mathbf{v}}}(\tau) d \tau
$$

where $\overline{\overline{\mathbf{v}}}(t)=\mathbf{Q}^{-1} \overline{\overline{\mathbf{u}}}(t)$ and $\mathbf{g}=\mathbf{Q}^{-1} \mathbf{h}$.

Note that $\mathbf{Q}$ satisfies the property $\mathbf{Q}^{H}=\mathbf{Q}^{-1}$. Also, $\Phi(0)$ can be expressed as the average eigenvalue $\Phi(0)=$ $\frac{1}{L} \sum_{l=0}^{L-1} \lambda_{l}=\bar{\lambda}$ since trace $\{\boldsymbol{\Phi}\}=L \Phi(0)=\sum_{l=0}^{L-1} \lambda_{l}$.

From (27), each element of $\overline{\overline{\mathbf{v}}}(t)$, i.e., $\overline{\bar{v}}_{l}(t)$, satisfies the integral equation

$\overline{\bar{v}}_{l}(t)=g_{l}-\mu \lambda_{l} \alpha \int_{0}^{t} e^{-\alpha(t-\tau)} \overline{\bar{v}}_{l}(\tau) d \tau, l=0,1, \cdots, L-1$,

where $g_{l}$ is the $l$-th element of $\mathbf{g}$.

The solution of $\overline{\bar{v}}_{l}(t)$ can be easily obtained through Laplace transform. That is, by performing Laplace transform on both sides of (28) and applying Laplace transform's convolution property, we have $\bar{V}_{l}(s)=g_{l} \frac{1}{s}-\mu \lambda_{l} \frac{\alpha}{s+\alpha} \overline{\bar{V}}_{l}(s)$, from which $\overline{\bar{V}}_{l}(s)$, the Laplace transform of $\overline{\bar{v}}_{l}(t)$, is solved as $\overline{\bar{V}}_{l}(s)=$ $\frac{g_{l} \frac{1}{s}}{1+\mu \lambda_{l} \frac{\alpha}{s+\alpha}}=\frac{(s+\alpha) g_{l}}{s^{2}+\left(1+\mu \lambda_{l}\right) \alpha s}=\frac{g_{l}}{1+\mu \lambda_{l}} \frac{1}{s}+\frac{\mu \lambda_{l}}{1+\mu \lambda_{l}} \frac{g_{l}}{s+\left(1+\mu \lambda_{l}\right) \alpha}$. Taking the inverse Laplace transform on $\overline{\bar{V}}_{l}(s), \overline{\bar{v}}_{l}(t)$ is solved as

$$
\overline{\bar{v}}_{l}(t)=\frac{g_{l}}{1+\mu \lambda_{l}}+\frac{\mu \lambda_{l} g_{l}}{1+\mu \lambda_{l}} e^{-\left(1+\mu \lambda_{l}\right) \alpha t}, t>0 .
$$

Consequently, $\overline{\overline{\mathbf{u}}}(t)$ is solved as

$$
\begin{aligned}
\overline{\overline{\mathbf{u}}}(t) & =\mathbf{Q} \overline{\overline{\mathbf{v}}}(t) \\
& =\mathbf{Q} \operatorname{diag}\left\{\frac{1}{1+\mu \lambda_{l}}+\frac{\mu \lambda_{l}}{1+\mu \lambda_{l}} e^{-\left(1+\mu \lambda_{l}\right) \alpha t}\right\} \mathbf{g} \\
& =\mathbf{Q} \operatorname{diag}\left\{\frac{1}{1+\mu \lambda_{l}}+\frac{\mu \lambda_{l}}{1+\mu \lambda_{l}} e^{-\left(1+\mu \lambda_{l}\right) \alpha t}\right\} \mathbf{Q}^{-1} \mathbf{h}
\end{aligned}
$$

where $\operatorname{diag}\{\cdot\}$ denotes the diagonal matrix with the $l$-th diagonal element specified as a function of $l$. 
From (12) and (30), the expectation and time averaging of $w_{l}(t)$ can be finally expressed as

$$
\begin{aligned}
\overline{\overline{\mathbf{w}}}(t) & =\bar{E}\{\mathbf{w}(t)\} \\
& =\operatorname{diag}\left\{e^{-j 2 \pi f_{c} T l}\right\} \\
& \cdot \mathbf{Q} \operatorname{diag}\left\{\frac{\mu \lambda_{l}}{1+\mu \lambda_{l}}\left[1-e^{-\left(1+\mu \lambda_{l}\right) \alpha t}\right]\right\} \mathbf{Q}^{-1} \mathbf{h} .
\end{aligned}
$$

We see that, as $t \rightarrow \infty, \quad \overline{\overline{\mathbf{w}}}(t)$ converges to $\operatorname{diag}\left\{e^{-j 2 \pi f_{c} T l}\right\} \mathbf{Q} \operatorname{diag}\left\{\frac{\mu \lambda_{l}}{1+\mu \lambda_{l}}\right\} \mathbf{Q}^{-1} \mathbf{h}$.

\section{Convergence of Residual Interference Power}

Furthermore, the normalized residual interference power can be evaluated as shown in (32) on top of next page. In deriving (32), the principle of orthogonality (22) is applied.

Assuming that $u_{l}(t), l=0,1, \cdots, L-1$, are independent to each other, (32) can be further expressed as

$$
\begin{aligned}
& P_{R I}(t) \\
& =\frac{1}{2} \varepsilon^{2}+\frac{1}{2} \bar{E}\left\{\sum_{l=0}^{L-1} \sum_{l^{\prime}=0, l^{\prime} \neq l}^{L-1} u_{l}^{*}(t) \Phi\left(\left(l-l^{\prime}\right) T\right) u_{l^{\prime}}(t)\right. \\
& \left.+\Phi(0) \sum_{l=0}^{L-1}\left|u_{l}(t)\right|^{2}\right\} \\
& =\frac{1}{2} \varepsilon^{2}+\frac{1}{2} \sum_{l=0}^{L-1} \sum_{l^{\prime}=0, l^{\prime} \neq l}^{L-1} \overline{\bar{u}}_{l}^{*}(t) \Phi\left(\left(l-l^{\prime}\right) T\right) \overline{\bar{u}}_{l^{\prime}}(t) \\
& +\frac{1}{2} \Phi(0) \sum_{l=0}^{L-1} \overline{\bar{u}}_{l}^{2}(t) \\
& =\frac{1}{2} \varepsilon^{2}+\frac{1}{2} \overline{\overline{\mathbf{u}}}^{H}(t)\left(\mathbf{\Phi}-\bar{\lambda} \mathbf{I}_{L}\right) \overline{\overline{\mathbf{u}}}(t)+\frac{1}{2} \bar{\lambda} \sum_{l=0}^{L-1} \overline{\bar{u}}_{l}^{2}(t)
\end{aligned}
$$

where $\overline{\bar{u}}_{l}^{2}(t)=\bar{E}\left\{\left|u_{l}(t)\right|^{2}\right\}$ is the mean squared value of $u_{l}(t)$ and $\mathbf{I}_{L}$ stands for the identity matrix of order $L$.

Since $\overline{\bar{u}}_{l}^{2}(t) \neq\left|\overline{\bar{u}}_{l}(t)\right|^{2}$, we cannot obtain $\overline{\bar{u}}_{l}^{2}(t)$ from (30) directly. However, from (25), a differential equation that $\sum_{l=0}^{L-1} \overline{\bar{u}}_{l}^{2}(t)$ satisfies can be obtained as

$$
\begin{aligned}
& \frac{d \sum_{l=0}^{L-1} \overline{\bar{u}}_{l}^{2}(t)}{d t}+2(1+\mu \bar{\lambda}) \alpha \sum_{l=0}^{L-1} \overline{\bar{u}}_{l}^{2}(t) \\
& =2 \alpha \operatorname{Re}\left\{\overline{\overline{\mathbf{u}}}^{H}(t) \mathbf{h}\right\}-2 \mu \alpha \overline{\overline{\mathbf{u}}}^{H}(t)\left(\boldsymbol{\Phi}-\bar{\lambda} \mathbf{I}_{L}\right) \overline{\overline{\mathbf{u}}}(t)
\end{aligned}
$$

and the solution can be derived accordingly as shown in (35) on next page (see Appendix B).

Substituting (30) and (35) into (33), we obtain $P_{R I}(t)$ as shown in (36) on next page. As $t \rightarrow \infty$, the normalized residual interference power converges to

$$
P_{R I}=\frac{1}{2} \varepsilon^{2}+\frac{1}{2} \mathbf{h}^{H} \mathbf{Q} \operatorname{diag}\left\{\frac{\lambda_{l}}{\left(1+\mu \lambda_{l}\right)^{2}}\right\} \mathbf{Q}^{-1} \mathbf{h} .
$$

We see that the steady normalized residual interference power is determined by the normalized modelling error, the LNA gain and the autocorrelation function of the transmitted signal.
Finally, from (24) the normalized interference power at RF can be expressed as

$$
P_{I}=\frac{1}{2} \varepsilon^{2}+\frac{1}{2} \mathbf{h}^{H} \boldsymbol{\Phi} \mathbf{h}
$$

and hence the interference suppression ratio is obtain as

$$
I S R=\frac{\varepsilon^{2}+\mathbf{h}^{H} \mathbf{Q} \operatorname{diag}\left\{\frac{\lambda_{l}}{\left(1+\mu \lambda_{l}\right)^{2}}\right\} \mathbf{Q}^{-1} \mathbf{h}}{\varepsilon^{2}+\mathbf{h}^{H} \mathbf{\Phi} \mathbf{h}} .
$$

It is easily seen that if there is no modelling error and the autocorrelation matrix has the same eigenvalue $\lambda_{0}=\lambda_{1}=$ $\ldots \lambda_{L-1}=\bar{\lambda}=A^{2}$ as in the ideal autocorrelation case, the ISR approaches $\frac{1}{\left(1+\mu A^{2}\right)^{2}}$, which is consistent with the cyclostationary analysis performed in Section III.

\section{Simulation AND EXPERIMENTAL RESUlts}

To verify the analytical results presented in Sections III and IV, numerical simulation and experimental validation are performed and the results are provided as follows.

\section{A. ALMS Loop Gain Calibration}

Given the multiplier dimensional constant product $K_{1} K_{2}$, the ALMS loop gain is determined by the LNA gain and the transmitted signal power as $\mu A^{2}=\mu \frac{V_{X}^{2}}{K_{1} K_{2}}$. The RMS voltage $V_{X}$ of the baseband signal $X(t)$ can be calculated through the RF signal power $P_{t}$ measured at the output of the HPA with load $R$, i.e., $V_{X}=\sqrt{2 R P_{t}}$. Also note that the LNA gain is $2 \mu$ and the loop gain can be also expressed as $\mu A^{2}=2 \mu \frac{V_{X}^{2}}{2 K_{1} K_{2}}=$ $2 \mu \frac{V_{x}^{2}}{K_{1} K_{2}}$ where $V_{x}=\sqrt{R P_{t}}$ is the RMS voltage of the RF signal $x(t)$.

Since the power is evaluated with $1 \Omega$ load in the above theoretical analysis, we need to add $17 \mathrm{~dB}(=10 \log (50))$ to convert a power measurement with $R=50 \Omega$ load to the one with $1 \Omega$ load.

\section{B. Numerical Simulation}

The numerical simulation uses a single carrier system with QPSK modulation and symbol duration $T_{s}=5 \mathrm{nS}$. The pulse shaping filter is an RRC filter with roll-off factor $\beta=0.25$ and hence the stationary autocorrelation function is a raisedcosine pulse. The carrier frequency is $f_{c}=2.4 \mathrm{GHz}$. All RF signals are converted into discrete signals with sampling period of $0.05 \mathrm{nS}$. The multi-tap adaptive filter has 8 taps when spaced at $T_{s}$ and 16 taps when spaced at $\frac{T_{s}}{2}$ so that the maximum interference channel delay is $8 T_{s}=40 \mathrm{nS}$. The LPF parameter for the ALMS loop is selected as $\alpha T_{s}=1.7655 \times$ $10^{-5}$ based on ISRLB $=10^{-13}$ using (20). The multiplier dimensional constants are selected as $K_{1} K_{2}=0.001 V^{2}$. The transmitted signal power is set to $0 \mathrm{dBm}$ with $50 \Omega$ load and hence $A=10$ or $20 \mathrm{~dB}$. We also set the self-interference power to $25 \mathrm{~dB}$ lower than the transmitted signal power and the combined received signal from remote transmitter plus thermal noise power to $60 \mathrm{~dB}$ lower than the self-interference power.

Two scenarios of interference channel are simulated to investigate the impacts of transmitted signal autocorrelation 


$$
\begin{aligned}
P_{R I}(t) & =\frac{1}{K_{1} K_{2}} \bar{E}\left\{[z(t)-y(t)]^{2}\right\}=\frac{1}{K_{1} K_{2}} \bar{E}\left\{\left[R e\left\{\left[Z(t)-\sum_{l=0}^{L-1} h_{l}^{*} X(t-l T)+\sum_{l=0}^{L-1} u_{l}^{*}(t) X(t-l T)\right] e^{j 2 \pi f_{c} t}\right\}\right]^{2}\right\} \\
& =\frac{1}{2 K_{1} K_{2}} \bar{E}\left\{\left|Z(t)-\sum_{l=0}^{L-1} h_{l}^{*} X(t-l T)+\sum_{=0}^{L-1} u_{l}^{*}(t) X(t-l T)\right|^{2}\right\} \\
& =\frac{1}{2 K_{1} K_{2}} \bar{E}\left\{\left|Z(t)-\sum_{l=0}^{L-1} h_{l}^{*} X(t-l T)\right|^{2}\right\}+\frac{1}{2 K_{1} K_{2}} \bar{E}\left\{\left|\sum_{=0}^{L-1} u_{l}^{*}(t) X(t-l T)\right|^{2}\right\} \\
& =\frac{1}{2} \varepsilon^{2}+\frac{1}{2 K_{1} K_{2}} \bar{E}\left\{\sum_{l=0}^{L-1} u_{l}^{*}(t) X(t-l T) \sum_{l^{\prime}=0}^{L-1} u_{l^{\prime}}(t) X^{*}\left(t-l^{\prime} T\right)\right\} \\
& =\frac{1}{2} \varepsilon^{2}+\frac{1}{2 K_{1} K_{2}} \bar{E}\left\{\sum_{l=0}^{L-1} \sum_{l^{\prime}=0}^{L-1} u_{l}^{*}(t) \bar{E}\left\{X(\tau-l T) X^{*}\left(\tau-l^{\prime} T\right)\right\} u_{l^{\prime}}(t)\right\} \\
& =\frac{1}{2} \varepsilon^{2}+\frac{1}{2} \bar{E}\left\{\sum_{l=0}^{L-1} \sum_{l^{\prime}=0}^{L-1} u_{l}^{*}(t) \Phi\left(\left(l-l^{\prime}\right) T\right) u_{l^{\prime}}(t)\right\} \\
& \left.+\frac{1}{\left(1+\mu \lambda_{l}\right)^{2}}+\frac{2 \mu \lambda_{l} \bar{\lambda}}{\left(1+\mu \lambda_{l}\right)^{2}} e^{-\left(1+\mu \lambda_{l}\right) \alpha t}+\frac{\mu^{2} \lambda_{l}^{2} \bar{\lambda}}{\left(1+\mu \lambda_{l}\right)^{2}} e^{-2\left(1+\mu \lambda_{l}\right) \alpha t}-\bar{\lambda} e^{-2(1+\mu \bar{\lambda}) \alpha t}\right\} \mathbf{Q}^{-1} \mathbf{h} \\
\sum_{l=0}^{L-1} & \bar{u}_{l}^{2}(t)=\frac{1}{2} \mathbf{h}^{H} \mathbf{Q} \operatorname{diag}\left\{\frac{\lambda_{l}}{\left(1+\mu \lambda_{l}\right)^{2}}+\frac{2 \mu \lambda_{l}^{2}}{\left(1+\mu \lambda_{l}\right)^{2}} e^{-\left(1+\mu \lambda_{l}\right) \alpha t}+\frac{\mu^{2} \lambda_{l}^{3}}{\left(1+\mu \lambda_{l}\right)^{2}} e^{-2\left(1+\mu \lambda_{l}\right) \alpha t}-\bar{\lambda} e^{-2(1+\mu \bar{\lambda}) \alpha t}\right\} \mathbf{Q}^{-1} \mathbf{h} \\
P_{R I}(t) & =\frac{1}{2} \varepsilon^{2}+\frac{1}{2} \mathbf{h}^{H} \mathbf{Q} \operatorname{diag}\left\{\frac{1}{\left(1+\mu \lambda_{l}\right)^{2}}+\frac{2 \mu \lambda_{l}}{\left(1+\mu \lambda_{l}\right)^{2}} e^{-\left(1+\mu \lambda_{l}\right) \alpha t}+\frac{\left(\mu \lambda_{l}\right)^{2}}{\left(1+\mu \lambda_{l}\right)^{2}} e^{-2\left(1+\mu \lambda_{l}\right) \alpha t}-e^{-2(1+\mu \bar{\lambda}) \alpha t}\right\} \mathbf{Q}^{-1} \mathbf{h}
\end{aligned}
$$

on the ALMS loop convergence performance and the interference channel modeling error on the residual interference power. For the first scenario, the interference channel is selected as $h(t)=10^{\frac{-25}{20}}\left\{\left[\frac{\sqrt{2}}{2}-0.5 j\right] \delta(t)-0.4 \delta\left(t-T_{s}\right)+\right.$ $\left.0.3 \delta\left(t-3 T_{s}\right)\right\}=[0.039764-0.028117 j] \delta(t)-0.022494 \delta(t-$ $\left.T_{s}\right)+0.016870 \delta\left(t-3 T_{s}\right)$, which means that the delays of the reflected paths are all integer multiples of $T_{s}$. The normalized interference power ( $1 \Omega$ load) can be found as $10^{\frac{-25+17-30}{10}} / K_{1} K_{2}=0.1581$. Two tap spacings of the multitap adaptive filter, $T_{s}$ and $\frac{T_{s}}{2}$, are tested to show the impact of autocorrelation on convergence performance. Fig. 6 shows the modelled impulse responses of the interference channel with $T_{s}$ spacing and $\frac{T_{s}}{2}$ spacing respectively. For the $T_{s}$ spacing case, the modelling error is 0 , whereas for the $\frac{T_{s}}{2}$ spacing case, the normalized modelling error is $8.3507 \times 10^{-10}$. Fig. 7 shows the convergence curves of the first tap coefficient $w_{0}(t)$ after averaging over 100 realizations when the LNA gain is set to $26 \mathrm{~dB}$ (i.e., $\mu=10$ ). We see that for both cases the simulated results and the theoretical ones obtained using (31) almost coincide at macro scale. The convergence speed is slightly slower for the $\frac{T_{s}}{2}$ spacing case. At micro scale, the simulated $\bar{w}_{0}(t)$ and $\overline{\bar{w}}_{0}(t)$ for the $T_{s}$ spacing case are shown in the inset. The fast variations of $\bar{w}_{0}(t)$ at period $T_{s}$ due to the cyclostationary property are clearly seen. However, it also demonstrates some slow variations which are not captured by the model expressed in (13) due to the independence assumption for analysis simplicity. This leaves some room for future research on more accurate mathematical models for ALMS loop analysis and hence the ISRLB can be further refined.

Fig. 8 shows the simulated convergence curves of the residual interference power and the corresponding theoretical ones obtained using (36) for the ALMS loop with $T_{s}$ spacing and $\frac{T_{s}}{2}$ spacing respectively. We see that, because the autocorrelation is zero at integer multiples of $T_{s}$ offsets, the ALMS loop with $T_{s}$ spacing converges faster than that with $\frac{T_{s}}{2}$ spacing. At $\mu=10$, the normalized residual interference power at $t=5000 T_{s}$ is $1.7425 \times 10^{-7}$ and thus the ISR is $1.7425 \times 10^{-7} / 0.1581=1.1 \times 10^{-6}(-59.6 \mathrm{~dB})$ which is close to the theoretical value $\frac{1}{\left(1+\mu A^{2}\right)^{2}}=0.998 \times 10^{-6}$. With $\frac{T_{s}}{2}$ spacing, the ALMS loop convergence speed is slowed down due to the correlation between signals at different adaptive 


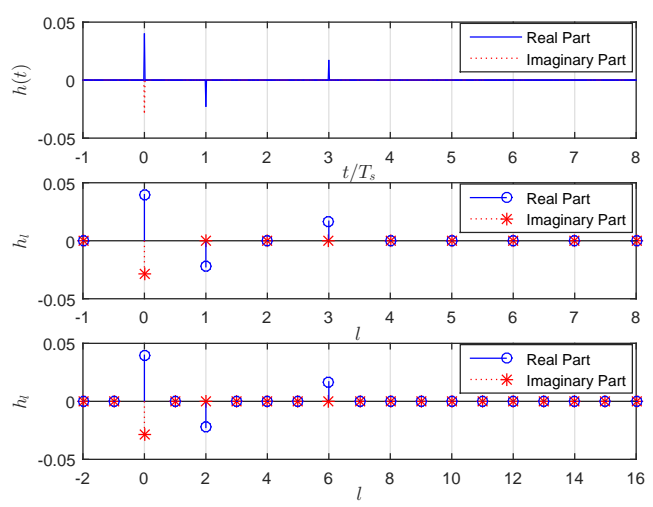

Fig. 6. $T_{s}$ spaced interference channel impulse response $h(t)$ (top) and modelled tap coefficients $h_{l}$ with $T_{s}$ spacing (middle) and $\frac{T_{s}}{2}$ spacing (bottom) respectively.

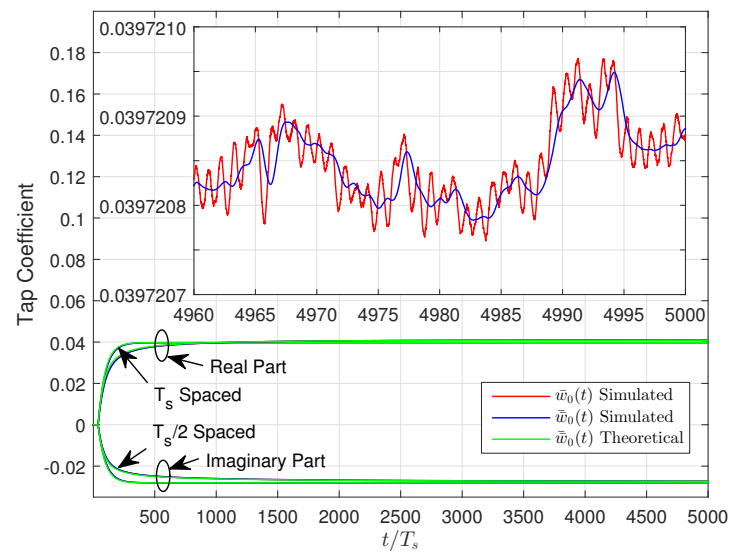

Fig. 7. Simulated and theoretical convergence curves for the first tap weighting coefficient of the ALMS loop with $T_{s}$ spacing and $\frac{T_{s}}{2}$ spacing respectively under the interference channel shown in Fig. 6.

filter taps. With larger LNA gain $46 \mathrm{~dB}$ (i.e., $\mu=100$ ), the convergence speed is increased and the residual interference power is lowered. However, we also see a gap between the simulated and theoretical residual interference powers for the case with $T_{s}$ spacing. This is because the impact of the signal from remote transmitter as well as the receiver noise on the ALMS loop convergence becomes more significant when the ISR is very low (lower than $10^{-8}$ in this case).

For the second scenario, the interference channel is selected as $h(t)=[0.039764-0.028117 j] \delta(t)-0.022494 \delta\left(t-0.9 T_{s}\right)+$ $0.016870 \delta\left(t-3.3 T_{s}\right)$ to simulate a general condition that the reflected paths can have arbitrary delays. Though the amplitude response of each path is the same as that in previous scenario, the normalized interference power is now calculated as 0.1428 due to the signal correlation between different paths. The modelled impulse responses of the interference channel with $T_{s}$ spacing and $\frac{T_{s}}{2}$ spacing respectively are shown in Fig. 9. In this case, the normalized channel modelling error with $T_{s}$ spacing is as large as 0.005 , whereas it is only $1.0163 \times 10^{-9}$ with $\frac{T_{s}}{2}$ spacing. This is easily understandable from the Nyquist sampling theorem. Since the signal bandwidth is larger than $\frac{1}{T_{s}}$ due to RRC pulse shaping, the interference channel model with $T_{s}$ spacing can not accurately represent a

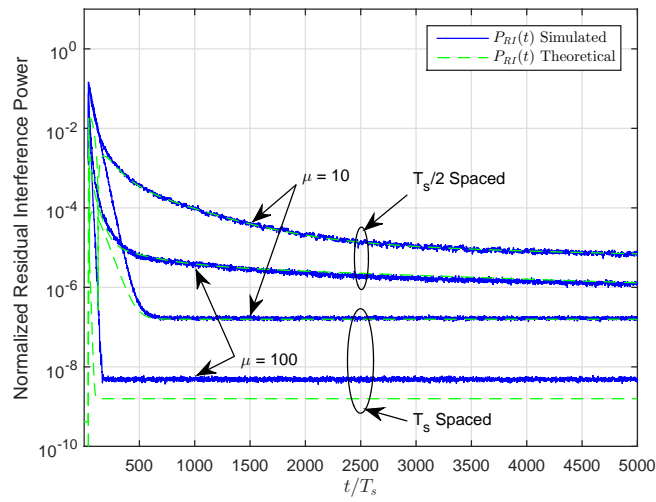

Fig. 8. Simulated and theoretical convergence curves for residual interference power of the ALMS loop with $T_{s}$ spacing and $\frac{T_{s}}{2}$ spacing respectively under the interference channel shown in Fig. 6.

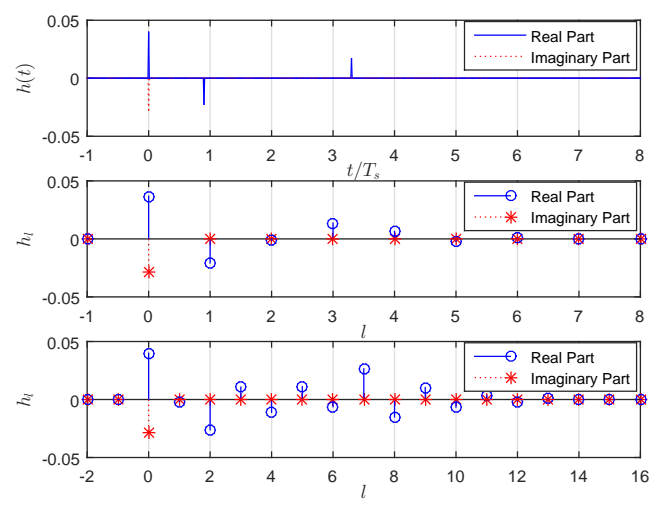

Fig. 9. Arbitrary interference channel impulse response $h(t)$ (top) and modelled tap coefficients $h_{l}$ with $T_{s}$ spacing (middle) and $\frac{T_{s}}{2}$ spacing (bottom) respectively.

general channel due to signal spectral aliasing.

The modelling error has significant impact on the residual interference power. As shown in Fig. 10, the ALMS loop with $T_{s}$ spacing can only achieve a normalized residual interference power of about $2.5 \times 10^{-3}$, whereas with $\frac{T_{s}}{2}$ spacing the ALMS loop still achieves similar levels of self-interference cancellation to those shown in Fig. 8.

\section{Experimental Validation}

The experimental setup is shown Fig. 11, where the RF frontend is composed of two $2.4 \mathrm{GHz}$ single-chip CMOS RF IC evaluation boards (RFX2401C) with respective Tx and Rx antennas. One board is set to Tx mode and the other to $\mathrm{Rx}$ mode. The Tx/Rx antenna spacing is $14 \mathrm{~cm}$. The transmitted baseband signal is generated burst by burst using a high speed FPGA development platform. Each burst consists of 1247 single carrier QPSK modulated random symbols with a symbol rate $50 \mathrm{Msps}$ (or $T_{s}=20 \mathrm{nS}$ ) and pulse shaped by a 0.25 roll-off RRC filter. The baseband signal is sampled at $2.5 \mathrm{Gsps}$ and shifted to $58 \mathrm{MHz}$ intermediate frequency (IF) digitally by the FPGA platform. The digital IF signal is finally filtered by a bandpass filter (CBPFS-2441) to produce 


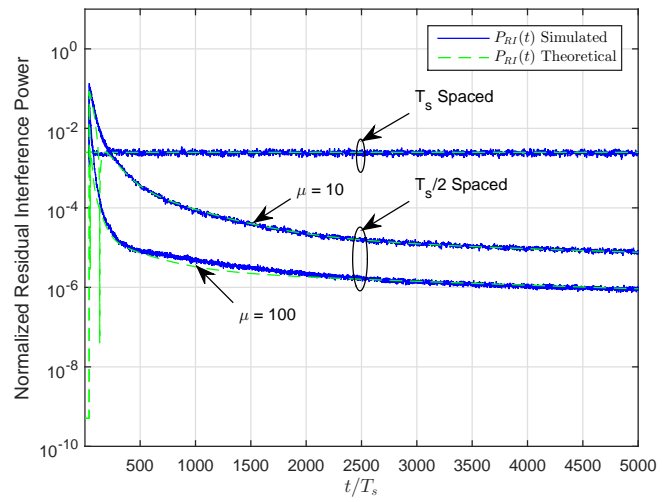

Fig. 10. Simulated and theoretical convergence curves for residual interference power of the ALMS loop with $T_{s}$ spacing and $\frac{T_{s}}{2}$ spacing respectively under the interference channel shown in Fig. 9.

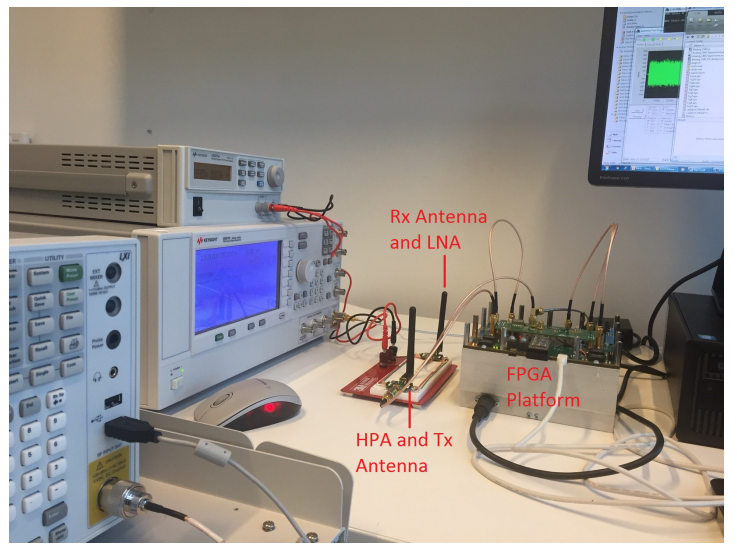

Fig. 11. Experimental setup with single-chip CMOS RF IC evaluation boards and an FPGA development platform.

the transmitted RF signal with center frequency $2.442 \mathrm{GHz}$. The signal amplitude is adjusted such that the RF signal power after HPA is measured as $0 \mathrm{dBm}(50 \Omega$ load). The received $\mathrm{RF}$ signal power is measured as $-14.5 \mathrm{dBm}$ at the $\mathrm{Rx}$ antenna and $-1 \mathrm{dBm}$ after the LNA, which means that the LNA gain is $13.5 \mathrm{~dB}$ (or $\mu=2.3658$ ). Both transmitted and received RF signal bursts are also captured by the FPGA development platform for further processing.

The purpose of this experiment is to validate the theoretical analysis on the ALMS loop performance with a practical interference channel and demonstrate the impact of the tap spacing and the number of taps of the adaptive filter on the residual interference power. Therefore, we assume that the components of the adaptive filter are ideal without I/Q imbalance. The multiplier dimensional constants are selected as $K_{1} K_{2}=0.001 V^{2}$ so that $A=10$ or $20 \mathrm{~dB}$. We also selected the RC constant of the LPF as $\alpha T_{s}=0.0883$ based on ISRLB $=10^{-9}$ so that the ALMS loop can converge quickly in every signal burst.

Fig. 12 shows the interference channel estimated by performing cross-correlation of the received signal burst with the transmitted signal burst and its 90 degree shifted versions as well as the weighting coefficients of the ALMS loop with $T_{s}$ spacing and $\frac{T_{s}}{2}$ spacing respectively converged at the end
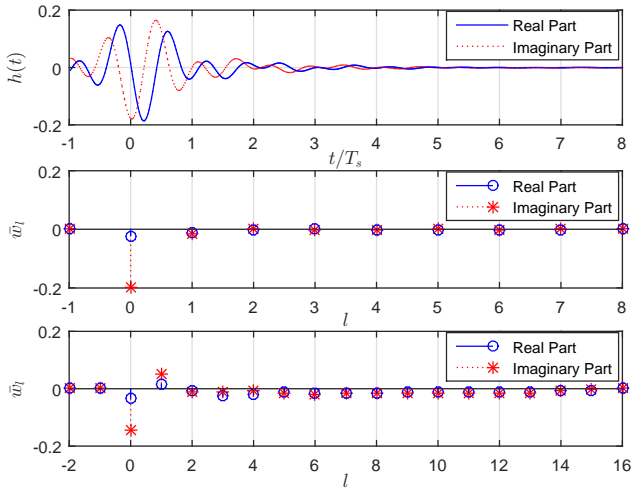

Fig. 12. Measured interference channel impulse response $h(t)$ (top) and converged weighting coefficients $\bar{w}_{l}$ with $T_{s}$ spacing (middle) and $\frac{T_{s}}{2}$ spacing (bottom) respectively.

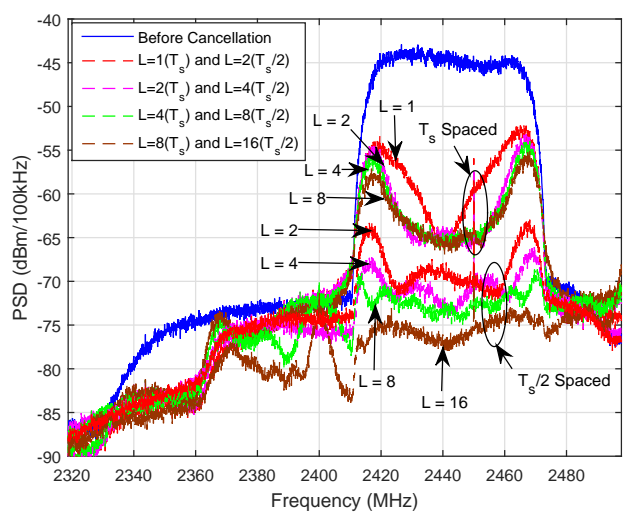

Fig. 13. Measured PSDs before and after self-interference cancellation with different tap spacings and numbers of taps in the adaptive filter.

of a signal burst. All the estimates are averaged over 100 signal bursts. We see that with this experimental setup the interference channel has major reflected paths clustered near the direct path, but the reflected paths are not resolvable since the path delays are much less than the symbol duration. The $\frac{T_{s}}{2}$ spaced adaptive filter better models the interference channel, as further verified by the measured power spectral densities (PSDs) shown in Fig. 13. We see that with $\frac{T_{s}}{2}$ spaced adaptive filter 26 to $33 \mathrm{~dB}$ self-interference cancellation is achieved even with the small LNA gain, whereas with $T_{s}$ spaced adaptive filter only a maximum of $20 \mathrm{~dB}$ cancellation is obtained at the center of the signal band. Given a tap spacing, the selfinterference cancellation performance is generally improved as the number of taps increases. However, it is also of interest to see that the $\frac{T_{s}}{2}$ spaced adaptive filter with only 2 taps performs better than the $T_{s}$ spaced one with 8 taps.

\section{Comparison with Existing Methods}

Finally we compare the architecture and performance of the proposed RF self-interference cancellation circuit with those of some existing ones $[12,13,19,21]$ as summarized in Table I on top of next page. It is easily seen that our proposed method is architecturally simpler since the tap weight 
TABLE I

COMPARISON WITH EXISTING RF CANCELLATION CIRCUITS

\begin{tabular}{|l|l|l|l|l|l|}
\hline \hline & Circuit Architecture & Down-Conversion & Tap Weight Control & ISR (dB) & Comment \\
\hline \hline$[12]$ & Multi-tap filter & - & Additional digital algorithm & -30 & $\begin{array}{l}\text { Experimental (8 taps) } \\
\text { Experimental (16 taps) }\end{array}$ \\
\hline$[13]$ & $\begin{array}{l}\text { Multi-tap filter with } \\
\text { phase shifters }\end{array}$ & Required & Integrator + 2 multipliers per tap & -56 & Simulation (3 taps, 2 echo paths) \\
\hline$[19]$ & I/Q multi-tap filter & Required & Integrator + 4 multipliers per tap & -33 & $\begin{array}{l}\text { Experimental (bandwidth 20 MHz) } \\
\text { Experimental (bandwidth 100 MHz) }\end{array}$ \\
\hline$[21]$ & I/Q multi-tap filter & Required & LPF + 4 multipliers per tap & -18 & -39.2 \\
& & & $\begin{array}{l}\text { Experimental (bandwidth 5 MHz) } \\
\text { Experimental (bandwidth 10 MHz) }\end{array}$ \\
\hline Proposed & I/Q multi-tap filter & Not required & LPF + 2 multipliers per tap & -59.4 & $\begin{array}{l}\text { Simulation (loop gain 30 dB ) } \\
\text { Experimental (loop gain 23.7 dB ) }\end{array}$ \\
\hline
\end{tabular}

control is implemented at RF rather than baseband. Both our simulation and experimental results confirm that the proposed method is able to approach the cancellation performance as expected by the theoretical analysis.

\section{Conclusions}

We have shown through cyclostationary analysis that there is always an irreducible residual interference after selfinterference cancellation using the proposed multi-tap adaptive filter with ALMS loop. Based on the overall self-interference suppression requirement including further digital domain cancellation, the ALMS loop parameter can be determined according to the derived interference suppression ratio lower bound. The performance of the ALMS loop is also characterized through stationary analysis, which shows that the convergence speed and achievable interference suppression ratio of the ALMS loop are determined by the loop gain and the autocorrelation function of the transmitted signal. The interference channel modelling error with the adaptive filter also has impact on the residual interference power. Future work includes cyclostationary analysis for different signaling schemes such as multicarrier systems to derive the respective interference suppression ratio lower bounds and stationary analysis of the self-interference cancellation performance in presence of practical impairments such as I/Q imbalance in the ALMS loop circuits.

\section{APPENDiX A: SOLUTIONS OF $\bar{u}_{l}(t)$ FOR CyCLOSTATIONARY ANALYSIS}

Firstly, we convert the integral equation (16) into a differential equation. By re-writing (16) as $\bar{u}_{l}(t)=$ $h_{l}-\mu A^{2} \alpha e^{-\alpha t} \int_{0}^{t} e^{\alpha \tau}\left(\frac{2 \beta}{\pi} \cos \frac{2 \pi}{T_{s}} \tau+1\right) \bar{u}_{l}(\tau) d \tau$, taking derivation with respect to $t$ on both sides to have $\frac{d \bar{u}_{l}(t)}{d t}=\mu A^{2} \alpha^{2} e^{-\alpha t} \int_{0}^{t} e^{\alpha \tau}\left(\frac{2 \beta}{\pi} \cos \frac{2 \pi}{T_{s}} \tau+1\right) \bar{u}_{l}(\tau) d \tau-$ $\mu A^{2} \alpha e^{-\alpha t} e^{\alpha t}\left(\frac{2 \beta}{\pi} \cos \frac{2 \pi}{T_{s}} t+1\right) \bar{u}_{l}(t)=\alpha\left[h_{l}-\bar{u}_{l}(t)\right]-$ $\mu A^{2} \alpha\left(\frac{2 \beta}{\pi} \cos \frac{2 \pi}{T_{s}} t+1\right) \bar{u}_{l}(t)$, and rearranging the derivation result, we obtain the ordinary differential equation (ODE)

$$
\frac{d \bar{u}_{l}(t)}{d t}+\alpha\left[1+\mu A^{2}\left(\frac{2 \beta}{\pi} \cos \frac{2 \pi}{T_{s}} t+1\right)\right] \bar{u}_{l}(t)=\alpha h_{l} \text {. }
$$

The homogeneous form of the ODE can be expressed as

$$
U^{\prime}(t)+\alpha\left[1+\mu A^{2}\left(\frac{2 \beta}{\pi} \cos \frac{2 \pi}{T_{s}} t+1\right)\right] U(t)=0
$$

where $(\cdot)^{\prime}$ denotes the first order derivative for simplicity. By rearranging (41) as $\frac{U^{\prime}(t)}{U(t)}=-\alpha\left[1+\mu A^{2}\left(\frac{2 \beta}{\pi} \cos \frac{2 \pi}{T_{s}} t+1\right)\right]$, using the fact that $\int_{0}^{t} \frac{U^{\prime}(\tau)}{U(\tau)} d \tau=\ln U(t)-\ln U(0)$ to have $\ln U(t)=-\alpha \int_{0}^{t}\left[1+\mu A^{2}\left(\frac{2 \beta}{\pi} \cos \frac{2 \pi}{T_{s}} \tau+1\right)\right] d \tau+$ $\ln U(0)=-\left(1+\mu A^{2}\right) \alpha t-\mu A^{2} \alpha T_{s} \frac{\beta}{\pi^{2}} \sin \frac{2 \pi}{T_{s}} t+\ln U(0)$, we find the solution of $U(t)$ as

$$
U(t)=U(0) e^{-\left(1+\mu A^{2}\right) \alpha t-\mu A^{2} \alpha T_{s} \frac{\beta}{\pi^{2}} \sin \frac{2 \pi}{T_{s}} t} .
$$

Replacing $U(0)$ by a function $f(t)$, the solution of the nonhomogeneous ODE can be expressed as

$$
\bar{u}_{l}(t)=f(t) e^{-\left(1+\mu A^{2}\right) \alpha t-\mu A^{2} \alpha T_{s} \frac{\beta}{\pi^{2}} \sin \frac{2 \pi}{T_{s}} t} .
$$

Taking derivation on both sides of (43), we have

$$
\begin{aligned}
\frac{d \bar{u}_{l}(t)}{d t}= & f^{\prime}(t) e^{-\left(1+\mu A^{2}\right) \alpha t-\mu A^{2} \alpha T_{s} \frac{\beta}{\pi^{2}} \sin \frac{2 \pi}{T_{s}} t} \\
& +f(t) e^{-\left(1+\mu A^{2}\right) \alpha t-\mu A^{2} \alpha T_{s} \frac{\beta}{\pi^{2}} \sin \frac{2 \pi}{T_{s}} t} \\
& \cdot\left[-\left(1+\mu A^{2}\right) \alpha-\mu A^{2} \alpha \frac{2 \beta}{\pi} \cos \frac{2 \pi}{T_{s}} t\right] .
\end{aligned}
$$

Substituting (44) and (43) into (40), we have

$$
\begin{aligned}
& f^{\prime}(t) e^{-\left(1+\mu A^{2}\right) \alpha t-\mu A^{2} \alpha T_{s} \frac{\beta}{\pi^{2}} \sin \frac{2 \pi}{T_{s}} t} \\
& +f(t) e^{-\left(1+\mu A^{2}\right) \alpha t-\mu A^{2} \alpha T_{s} \frac{\beta}{\pi^{2}} \sin \frac{2 \pi}{T_{s}} t} \\
& \cdot\left[-\left(1+\mu A^{2}\right) \alpha-\mu A^{2} \alpha \frac{2 \beta}{\pi} \cos \frac{2 \pi}{T_{s}} t\right] \\
& +\alpha\left[1+\mu A^{2}\left(\frac{2 \beta}{\pi} \cos \frac{2 \pi}{T_{s}} t+1\right)\right] \\
& \cdot f(t) e^{-\left(1+\mu A^{2}\right) \alpha t-\mu A^{2} \alpha T_{s} \frac{\beta}{\pi^{2}} \sin \frac{2 \pi}{T_{s}} t}=\alpha h_{l}
\end{aligned}
$$

from which we obtain

$$
f^{\prime}(t)=\alpha h_{l} e^{\left(1+\mu A^{2}\right) \alpha t+\mu A^{2} \alpha T_{s} \frac{\beta}{\pi^{2}} \sin \frac{2 \pi}{T_{s}} t} .
$$

Thus $f(t)$ is determined as

$$
f(t)=\alpha h_{l} \int_{0}^{t} e^{\left(1+\mu A^{2}\right) \alpha \tau+\mu A^{2} \alpha T_{s} \frac{\beta}{\pi^{2}} \sin \frac{2 \pi}{T_{s}} \tau} d \tau+C
$$

where $\mathrm{C}$ is any constant, and hence the solution of $\bar{u}_{l}(t)$ is obtained as

$$
\begin{aligned}
\bar{u}_{l}(t)= & {\left[\alpha h_{l} \int_{0}^{t} e^{\left(1+\mu A^{2}\right) \alpha \tau+\mu A^{2} \alpha T_{s} \frac{\beta}{\pi^{2}} \sin \frac{2 \pi}{T_{s}} \tau} d \tau+C\right] } \\
& \cdot e^{-\left(1+\mu A^{2}\right) \alpha t-\mu A^{2} \alpha T_{s} \frac{\beta}{\pi^{2}} \sin \frac{2 \pi}{T_{s}} t} .
\end{aligned}
$$


Eq. (45) still involves an integral which is not convenient in use. However, by re-writing it as $\bar{u}_{l}(t)=$ $\left[\alpha h_{l} \int_{0}^{t} e^{-\left(1+\mu A^{2}\right) \alpha(t-\tau)} e^{\mu A^{2} \alpha T_{s} \frac{\beta}{\pi^{2}} \sin \frac{2 \pi}{T_{s}} \tau} d \tau+C e^{-\left(1+\mu A^{2}\right) \alpha t}\right]$ $\cdot e^{-\mu A^{2} \alpha T_{s} \frac{\beta}{\pi^{2}} \sin \frac{2 \pi}{T_{s}} t}$ and using the approximation $\int_{0}^{t} e^{-\left(1+\mu A^{2}\right) \alpha(t-\tau)} e^{\mu A^{2} \alpha T_{s} \frac{\beta}{\pi^{2}} \sin \frac{2 \pi}{T_{s}} \tau} d \tau \approx \frac{1}{\alpha\left(1+\mu A^{2}\right)}$ which is valid for $t>0$ and $\mu A^{2} \alpha T_{s} \frac{\beta}{\pi^{2}}<<1, \bar{u}_{l}(t)$ can be approximated as

$$
\bar{u}_{l}(t) \approx\left[\frac{h_{l}}{1+\mu A^{2}}+C e^{-\left(1+\mu A^{2}\right) \alpha t}\right] e^{-\mu A^{2} \alpha T_{s} \frac{\beta}{\pi^{2}} \sin \frac{2 \pi}{T_{s}} t} .
$$

From the initial condition $\bar{u}_{l}(0)=h_{l}$, which is easily seen from (16), we have $C=h_{l} \frac{\mu A^{2}}{1+\mu A^{2}}$ and finally obtain the closed-form approximate solution as shown in (17).

\section{APPENDiX B: SOLUTION OF $\sum_{l=0}^{L-1} \overline{\bar{u}}_{l}^{2}(t)$ FOR STATIONARY ANALYSIS}

Firstly, we convert the integral equation (25) into a differential equation using the same process as in Appendix A, resulting in

$$
\begin{aligned}
& \frac{d u_{l}(t)}{d t}=\alpha\left[h_{l}-u_{l}(t)\right]-\frac{\mu \alpha}{K_{1} K_{2}}\left[Z^{*}(t)-\sum_{l^{\prime}=0}^{L-1} h_{l^{\prime}} X^{*}\left(t-l^{\prime} T\right)\right. \\
& \left.+\sum_{l^{\prime}=0}^{L-1} u_{l^{\prime}}(t) X^{*}\left(t-l^{\prime} T\right)+S^{*}(t)+N^{*}(t)\right] X(t-l T) .
\end{aligned}
$$

Multiplying by $2 u_{l}^{*}(t)$ on both sides of (47), we have

$$
\begin{aligned}
& 2 u_{l}^{*}(t) \frac{d u_{l}(t)}{d t}=2 \alpha u_{l}^{*}(t) h_{l}-2 \alpha\left|u_{l}(t)\right|^{2}-\frac{2 \mu \alpha}{K_{1} K_{2}} u_{l}^{*}(t)\left[Z^{*}(t)\right. \\
& -\sum_{l^{\prime}=0}^{L-1} h_{l^{\prime}} X^{*}\left(t-l^{\prime} T\right)+\sum_{l^{\prime}=0}^{L-1} u_{l^{\prime}}(t) X^{*}\left(t-l^{\prime} T\right)+S^{*}(t) \\
& \left.+N^{*}(t)\right] X(t-l T)
\end{aligned}
$$

Using the equation $\frac{d|f(t)|^{2}}{d t}=2 \operatorname{Re}\left\{f^{*}(t) \frac{d f(t)}{d t}\right\}$ for any complex function $f(t)$, we have

$$
\begin{aligned}
& \frac{d\left|u_{l}(t)\right|^{2}}{d t}=2 \alpha \operatorname{Re}\left\{u_{l}^{*}(t) h_{l}\right\}-2 \alpha\left|u_{l}(t)\right|^{2}-\frac{2 \mu \alpha}{K_{1} K_{2}} \operatorname{Re}\left\{u_{l}^{*}(t)\right. \\
& \cdot\left[Z^{*}(t)-\sum_{l^{\prime}=0}^{L-1} h_{l^{\prime}} X^{*}\left(t-l^{\prime} T\right)+\sum_{l^{\prime}=0}^{L-1} u_{l^{\prime}}(t) X^{*}\left(t-l^{\prime} T\right)\right. \\
& \left.\left.+S^{*}(t)+N^{*}(t)\right] X(t-l T)\right\} .
\end{aligned}
$$

Performing ensemble expectation and time averaging on both sides of (49) and applying the principle of orthogonality (22) and independence assumptions as before, we have

$$
\begin{aligned}
& \frac{d \overline{\bar{u}}_{l}^{2}(t)}{d t}=2 \alpha \operatorname{Re}\left\{\overline{\bar{u}}_{l}^{*}(t) h_{l}\right\}-2 \alpha \overline{\bar{u}}_{l}^{2}(t)-2 \mu \alpha \\
& \cdot \operatorname{Re}\left\{\sum_{l^{\prime}=0, l^{\prime} \neq l}^{L-1} \overline{\bar{u}}_{l}^{*}(t) \Phi\left(\left(l-l^{\prime}\right) T\right) \overline{\bar{u}}_{l^{\prime}}(t)+\Phi(0) \overline{\bar{u}}_{l}^{2}(t)\right\} .
\end{aligned}
$$

Summing up all equations for $l=0,1, \cdots, L-1$, we have

$$
\begin{aligned}
& \frac{d \sum_{l=0}^{L-1} \overline{\bar{u}}_{l}^{2}(t)}{d t}=2 \alpha R e\left\{\sum_{l=0}^{L-1} \overline{\bar{u}}_{l}^{*}(t) h_{l}\right\}-2(1+\mu \Phi(0)) \alpha \\
& \cdot \sum_{l=0}^{L-1} \overline{\bar{u}}_{l}^{2}(t)-2 \mu \alpha \sum_{l=0}^{L-1} \sum_{l^{\prime}=0, l^{\prime} \neq l}^{L-1} \bar{u}_{l}^{*}(t) \Phi\left(\left(l-l^{\prime}\right) T\right) \overline{\bar{u}}_{l^{\prime}}(t) .
\end{aligned}
$$

Rearranging (51) and expressing the terms with summation in matrix form, we obtained the differential equation as shown in (34).

Denoting $b(t)=\sum_{l=0}^{L-1} \overline{\bar{u}}_{l}^{2}(t)$ and substituting (30) into (34), we have (52) as shown on top of next page. The solution of $b(t)$ can be also obtained through Laplace transform. Performing Laplace transform on both sides of (52), we have (53) as shown in the second equation on next page from which $B(s)$, the Laplace transform of $b(t)$, is solved as (54) shown in the third equation on next page. Taking the inverse Laplace transform on $B(s), b(t)$ is solved as shown in (35).

\section{REFERENCES}

[1] A. Sabharwal, P. Schniter, D. Guo, D. W. Bliss, S. Rangarajan, and R. Wichman, "In-band full-duplex wireless: challenges and opportunities," IEEE Journal on Selected Areas in Communications, Vol. 32, No. 9, September 2014, pp.1637 - 1651.

[2] M. Duarte and A. Sabharwal, "Full-duplex wireless communications using off-the-shelf radios: feasibility and first results," The Forty Fourth Asilomar Conference on Signals, Systems and Computers (ASILOMAR2010), Pacific Grove, CA, USA, 7 - 10 November 2010.

[3] M. Duarte, C. Dick, and A. Sabharwal, "Experiment-driven characterization of full-duplex wireless systems," IEEE Transactions on Wireless Communications, Vol. 11, No. 12, December 2012, pp. 4296 - 4307.

[4] T. A. Levanen, J. Pirskanen, T. Koskela, J. Talvitie, and M. Valkama, "Radio interface evolution towards $5 \mathrm{G}$ and enhanced local area communications," IEEE Access, Vol. 2, 2014, pp. 1005 - 1029.

[5] S. Han, C.-L. I, Z. Xu, C. Pan, and Z. Pan, "Full duplex: coming into reality in 2020?" IEEE 2014 Global Communications Conference (Globecom2014), Austin, TX, USA, 8 - 12 December 2014.

[6] X. Xie and X. Zhang, "Does full-duplex double the capacity of wireless networks?" IEEE 2014 International Conference on Computer Communications (INFOCOM2014), Totonto, Canada, 27 April - 2 May 2014.

[7] B. Debaillie, D.-J. van den Broek, C. Lavn, B. van Liempd, E. A. M. Klumperink, C. Palacios, J. Craninckx, B. Nauta, and A. Prssinen, "Ana$\log / \mathrm{RF}$ solutions enabling compact full-duplex radios," IEEE Journal on Selected Areas in Communications, Vol. 32, No. 9, September 2014, pp. $1662-1673$.

[8] L. Laughlin, M. A. Beach, K. A. Morris, and J. L. Haine, "Optimum single antenna full duplex using hybrid junctions," IEEE Journal on Selected Areas in Communications, Vol. 32, No. 9, September 2014, pp. $1653-1661$.

[9] J. I. Choi, M. Jain, K. Srinivasan, P. Levis, and S. Katti, "Achieving single channel, full duplex wireless communication." The 16th Annual International Conference on Mobile Computing andNetworking (Mobicom2010), Chicago, Illinois, USA, 20 - 24 September 2010.

[10] M. Jain, J. I. Choi, T. Kim, D. Bharadia, K. Srinivasan, S. Seth, P. Levis, S. Katti, and P. Sinha, "Practical, real-time, full duplex wireless," The 17th Annual International Conference on Mobile Computing and Networking (Mobicom2011), Las Vegas, Nevada, USA, 19 - 23 September 2011.

[11] S. Maddio, A. Cidronali, and G. Manes, "Real-time adaptive transmitter leakage cancelling in 5.8-GHz full-duplex transceivers," IEEE Transactions on Microwave Theory and Techniques, Vol. 63, No. 2, February 2015, pp. 509 - 519.

[12] D. Bharadia, E. McMillin, and S. Katti, "Full duplex radios," 2013 Annual Conference of ACM Special Interest Group on Data Communication (Sigcomm2013), Hong Kong, China, 12 - 16 August 2013.

[13] Y.-S. Choi and H. Shirani-Mehr, "Simultaneous transmission and reception: algorithm, design and system level performance," IEEE Transactions on Wireless Communications, Vol. 12, No. 12, December 2013, pp. $5992-6010$. 


$$
\begin{aligned}
& \frac{d b(t)}{d t}+2(1+\mu \bar{\lambda}) \alpha b(t)=2 \alpha \mathbf{h}^{H} \mathbf{Q} \operatorname{diag}\left\{\frac{1}{1+\mu \lambda_{l}}+\frac{\mu \lambda_{l}}{1+\mu \lambda_{l}} e^{-\left(1+\mu \lambda_{l}\right) \alpha t}\right\} \mathbf{Q}^{-1} \mathbf{h} \\
& -2 \mu \alpha \mathbf{h}^{H} \mathbf{Q} \operatorname{diag}\left\{\left(\lambda_{l}-\bar{\lambda}\right)\left(\frac{1}{1+\mu \lambda_{l}}+\frac{\mu \lambda_{l}}{1+\mu \lambda_{l}} e^{-\left(1+\mu \lambda_{l}\right) \alpha t}\right)^{2}\right\} \mathbf{Q}^{-1} \mathbf{h} \\
& =2 \alpha \mathbf{h}^{H} \mathbf{Q} \operatorname{diag}\left\{\frac{1}{1+\mu \lambda_{l}}+\frac{\mu \lambda_{l}}{1+\mu \lambda_{l}} e^{-\left(1+\mu \lambda_{l}\right) \alpha t}-\mu\left(\lambda_{l}-\bar{\lambda}\right)\left(\frac{1}{1+\mu \lambda_{l}}+\frac{\mu \lambda_{l}}{1+\mu \lambda_{l}} e^{-\left(1+\mu \lambda_{l}\right) \alpha t}\right)^{2}\right\} \mathbf{Q}^{-1} \mathbf{h} \\
& =2 \alpha \mathbf{h}^{H} \mathbf{Q} \operatorname{diag}\left\{\frac{1+\mu \bar{\lambda}}{\left(1+\mu \lambda_{l}\right)^{2}}+\frac{\mu \lambda_{l}\left(1-\mu \lambda_{l}+2 \mu \bar{\lambda}\right)}{\left(1+\mu \lambda_{l}\right)^{2}} e^{-\left(1+\mu \lambda_{l}\right) \alpha t}-\frac{\mu\left(\lambda_{l}-\bar{\lambda}\right)\left(\mu \lambda_{l}\right)^{2}}{\left(1+\mu \lambda_{l}\right)^{2}} e^{-2\left(1+\mu \lambda_{l}\right) \alpha t}\right\} \mathbf{Q}^{-1} \mathbf{h} \\
& s B(s)+2(1+\mu \bar{\lambda}) \alpha B(s)= \\
& 2 \alpha \mathbf{h}^{H} \mathbf{Q} \operatorname{diag}\left\{\frac{1+\mu \bar{\lambda}}{\left(1+\mu \lambda_{l}\right)^{2}} \frac{1}{s}+\frac{\mu \lambda_{l}\left(1-\mu \lambda_{l}+2 \mu \bar{\lambda}\right)}{\left(1+\mu \lambda_{l}\right)^{2}} \frac{1}{s+\left(1+\mu \lambda_{l}\right) \alpha}-\frac{\mu\left(\lambda_{l}-\bar{\lambda}\right)\left(\mu \lambda_{l}\right)^{2}}{\left(1+\mu \lambda_{l}\right)^{2}} \frac{1}{s+2\left(1+\mu \lambda_{l}\right) \alpha}\right\} \mathbf{Q}^{-1} \mathbf{h} \\
& B(s)=2 \alpha \mathbf{h}^{H} \mathbf{Q} \operatorname{diag}\left\{\frac{1+\mu \bar{\lambda}}{\left(1+\mu \lambda_{l}\right)^{2}} \frac{1}{s[s+2(1+\mu \bar{\lambda}) \alpha]}+\frac{\mu \lambda_{l}\left(1-\mu \lambda_{l}+2 \mu \bar{\lambda}\right)}{\left(1+\mu \lambda_{l}\right)^{2}} \frac{1}{\left[s+\left(1+\mu \lambda_{l}\right) \alpha\right][s+2(1+\mu \bar{\lambda}) \alpha]}\right. \\
& \left.-\frac{\mu\left(\lambda_{l}-\bar{\lambda}\right)\left(\mu \lambda_{l}\right)^{2}}{\left(1+\mu \lambda_{l}\right)^{2}} \frac{1}{\left[s+2\left(1+\mu \lambda_{l}\right) \alpha\right][s+2(1+\mu \bar{\lambda}) \alpha]}\right\} \mathbf{Q}^{-1} \mathbf{h} \\
& =\mathbf{h}^{H} \mathbf{Q} \operatorname{diag}\left\{\frac{1}{\left(1+\mu \lambda_{l}\right)^{2}} \frac{1}{s}+\frac{2 \mu \lambda_{l}}{\left(1+\mu \lambda_{l}\right)^{2}} \frac{1}{s+\left(1+\mu \lambda_{l}\right) \alpha}+\frac{\left(\mu \lambda_{l}\right)^{2}}{\left(1+\mu \lambda_{l}\right)^{2}} \frac{1}{s+2\left(1+\mu \lambda_{l}\right) \alpha}-\frac{1}{s+2(1+\mu \bar{\lambda}) \alpha}\right\} \mathbf{Q}^{-1} \mathbf{h}
\end{aligned}
$$

[14] D. Korpi, T. Huusari, Y.-S. Choi, L. Anttila, S. Talwar, and M. Valkama, "Full-duplex mobile device - pushing the limits," available: arxiv.org/pdf/1410.3191

[15] S. Karni and G. Zeng, "The analysis of the continuous-time LMS algorithm," IEEE Transactions on Acoustics, Speech, and Signal Processing, Vol. 37, No. 4. April 1989, pp. 595 - 597.

[16] A. Carusone and D. A. Johns, "Analogue adaptive filters: past and present," IEE Proceedings - Ciruits, Devices and Systems, Vol. 147 No. 1, Februaruy 2000 pp. $82-90$.

[17] J. E. Perez-Carmona, H. M. Perez-Meana and J. C. Sanchez-Garcia "Analog adaptive filter LMS algorithm using CMOS $0.5 \mathrm{~m}$ technology," WSEAS Transactions on Circuits and Systems, Vol. 7, No. 9, September 2008, pp. 839 - 848.

[18] D. Korpi, T. Riihonen, V. Syrjala, L. Anttila, M. Valkama, and R. Wichman, "Full-duplex transceiver system calculations: Analysis of ADC and linearity challenges," IEEE Transactions on Wireless Communications, Vol. 13, No. 7, July 2014, pp. 3821 - 3836.

[19] T. Huusari, Y.-S. Choi, P. Liikkanen, D. Korpi, S. Talwar, and M. Valkama, "Wideband self-adaptive RF cancellation circuit for full-duplex radio: Operating principle and measurements," in Proc. IEEE 81st Vehicular Technology Conference (VTC2015-Spring), Glasgow, Scotland, 11-14 May 2015.

[20] Y. Hua, Y. Ma, A. Gholian, Y. Li, A. C. Cirik, and P. Liang, "Radio self-interference cancellation by transmit beamforming, all-analog cancellation and blind digital tuning," Elsevier Signal Processing, Vol. 108 (2015), pp. $322-340$.

[21] S. Kim, Y. Jeon, G. Noh, Y.-O. Park, I. Kim, and H. Shin, "A 2.59-GHz RF self-interference cancellation circuit with wide dynamic range for in-band full-duplex radio," IEEE International Microwave Symposium (IMS2016), San Francisco, CA, 22 - 27 May 2016. 